\title{
Projective normality and syzygies of algebraic surfaces
}

\author{
Dedicated to David Eisenbud on his fiftieth birthday
}

By F.J. Gallego ${ }^{1}$ ) at Madrid and B.P. Purnaprajna at Lawrence

\begin{abstract}
In this work we develop new techniques to compute Koszul cohomology groups for several classes of varieties. As applications we prove results on projective normality and syzygies for algebraic surfaces. From more general results we obtain in particular the following:
\end{abstract}

(a) Mukai's conjecture (and stronger variants of it) regarding projective normality and normal presentation for surfaces with Kodaira dimension 0 , and uniform bounds for higher syzygies associated to adjoint linear series,

(b) effective bounds along the lines of Mukai's conjecture regarding projective normality and normal presentation for surfaces of positive Kodaira dimension, and,

(c) results on projective normality for pluricanonical models of surfaces of general type (recovering and strengthening results by Ciliberto) and generalizations of them to higher syzygies.

In addition, we also extend the above results to singular surfaces.

\section{Introduction}

In this article we develop new techniques to compute Koszul cohomology groups. Koszul cohomology is important because of its relation to Hodge Theory and to the computation of syzygies of projective varieties. In the present work we focus on the latter application. The topic of syzygies is interesting because it deals with the interplay between algebra and geometry: the algebra coming from the equations defining the variety and the geometry arising from the knowledge of what line bundles live on the variety. The earliest

*) Partially supported by DGES Grant PB 96-0659. 
result typical of this application we have in mind is the result of Castelnuovo, who showed that a curve of degree greater than $2 g$ has a normal homogeneous coordinate ring ( $g$ denotes the genus of the curve). He also proved that if the degree was greater than $2 g+1$, then the ideal of the curve was generated by quadratic equations. This result was rediscovered later by many people, among others Fujita, St. Donat, Mumford, Green, etc. Recently Mark Green threw new light on this connection between algebra and geometry by generalizing the study of homogeneous coordinate rings and ideals to the study of free resolutions. He linked the behavior of graded Betti numbers of the resolution of the homogeneous coordinate ring to the cohomology groups of certain vector bundles on the variety (see [G1], [G2] and [G3]; for a particularly nice introduction to the subject see also [L] and for the precise statement used in this article see Theorem 1.2). Green generalized Castelnuovo's result proving that if the degree of the curve is greater than $2 g+p$, then the resolution is in addition linear until the $p$ th stage. This property of the resolution is the so-called property $N_{p}$. Connection between algebra and geometry is better seen in the case of the canonical curve. Here there are classical results by Nöther and Petri on projective normality and normal presentation for canonical curves. The geometric part of the statements is summed up in the Clifford index of the curves. Green's conjecture for canonical curves generalizes Nöther and Petri's results, claiming that the shape of the free resolution of the canonical ring is determined by the Clifford index of the curve (precisely, if the Clifford index is $p+1$, then the resolution satisfies exactly the property $N_{p}$ ).

There are still many open questions regarding linear series on curves, but for surfaces and higher dimensional varieties the field is almost entirely open. Among the open questions for surfaces and higher dimensional varieties, the conjectures of Fujita on very ampleness and Mukai on higher syzygies of surfaces have attracted attention in recent years. Fujita conjectured that on an algebraic variety $X$ of dimension $n$, if $A$ is an ample line bundle on $X$, then $K_{X} \otimes A^{\otimes n+2}$ should be very ample, where $K_{X}$ denotes the canonical bundle on $X$. Mukai's conjecture says that if $S$ is a surface, $A$ is an ample line bundle on $S, L$ is a line bundle on $S$ equal to $K_{S} \otimes A^{\otimes n}$, and $n \geqq p+4$, then $L$ satisfies property $N_{p}$. This conjecture can be regarded as a two dimensional analogue of Green's theorem for curves. Indeed, Green's theorem can be interpreted as follows: any line bundle $L$ on a curve $C$ which is at least as positive as $K_{C} \otimes A^{\otimes p+3}$ satisfies property $N_{p}$, where $K_{C}$ is the canonical bundle of $C$ and $A$ is an ample line bundle on $C$. Fujita's conjecture has been proved for algebraic surfaces and it follows from a remarkable result of Reider (cf. [R]). For higher dimensional varieties some effective bounds have been obtained. Even though the bounds are far from what has been conjectured, they are considered an important step towards the goal of proving Fujita's conjecture. Mukai's conjecture has not yet been proved even for $p=0$. Some progress has been made by Butler for ruled varieties (see [Bu]), Kempf for Abelian varieties (see $[\mathrm{Ke}]$ ), and Ein and Lazarsfeld, who prove a beautiful, very general result on adjoint linear series associated to very ample line bundles (see [EL]). Y. Homma proved Mukai's conjecture for the case $p=0$ for elliptic ruled surfaces (see [H1] and [H2]). One of the things we do here is to prove Mukai's conjecture in certain cases and obtain effective bounds towards it for all surfaces.

In this article we pursue a new direction to study syzygies of algebraic surfaces. This direction can be summarized in the following meta-principle:

(0.1) If $L$ is the product of $(p+1)$ ample and base-point-free line bundles satisfying "certain cohomological" conditions, then $L$ satisfies the condition $N_{p}$. 
With the meta-principle as a guiding light, we obtain the following as corollaries of our more general results:

(1) We prove that Mukai's conjecture regarding projective normality and normal presentation is true, lowering Mukai's bound by one in the latter case, for all surfaces of Kodaira dimension 0 and show stronger variants of it (cf. Corollaries 2.6 and 4.5).

(2) We obtain a uniform bound along the line of Mukai's conjecture for higher syzygies associated to adjoint linear series for all surfaces of Kodaira dimension 0 .

(3) We find effective bounds along the lines of Mukai's conjecture regarding projective normality and normal presentation for surfaces of positive Kodaira dimension.

(4) We obtain results on projective normality, normal presentation and higher syzygies for pluricanonical models of surfaces of general type, recovering and strengthening results of Ciliberto (cf. [Ci]).

(5) We find effective bounds regarding projective normality and higher syzygies for multiples of ample bundles for regular surfaces of positive Kodaira dimension.

Result (3) can be interpreted as a higher syzygy analogue along the lines of Mukai's of the effective results of Demailly, Ein and Lazarsfeld regarding Fujita's conjecture. Result (5) is a higher syzygy analogue of the results of Siu and Fernández del Busto regarding effective Matsusaka's theorems on base-point-freeness and very ampleness. On the other hand, since an effective bound regarding Mukai's conjecture was obtained by Butler for ruled surfaces, results (1), (2) and (3), coupled with Butler's give the best bounds so far towards Mukai's conjecture for all surfaces.

Almost all known results on syzygies of algebraic surfaces (and several results on curves) fit into 0.1 . For example, the normal presentation of line bundles of degree greater than $2 g+1$ on curves, by Castelnuovo and others (see [GP1]), the result of Kempf referred to above (see Remark 4.6), and the result by Ein and Lazarsfeld. In [GP1], [GP2] and [GP 3] we show the validity of 0.1 for surfaces of Kodaira dimension $-\infty$ and K 3 surfaces. We show in the present article that 0.1 holds for all other surfaces of Kodaira dimension 0 and for adjoint linear series (more general than those involved in Mukai's conjecture) on surfaces of positive Kodaira dimension. We summarize here the results which give evidence of the above claims:

For surfaces of Kodaira dimension $-\infty$, the $(p+1)$-th power of an ample, free and nonspecial line bundle satisfies property $N_{p}$ ([GP2], Theorem 2.2, see also Lemma 2.8; our result is in fact more general as it is stated for surfaces with geometric genus 0 ). Theorem 1.3 of this paper generalizes this result and unifies among others Corollary 5.11 for surfaces of general type and Corollary 1.6 for Calabi-Yau threefolds. Moreover, in [GP1] and [GP2] we prove finer versions ([GP1], Theorem 4.2 and [GP2], Theorem 6.1) of the meta-principle for elliptic ruled surfaces, yielding among other things the fact that Mukai's conjecture regarding normal presentation holds for such surfaces. For anticanonical rational surfaces we also prove finer versions of 0.1 , in a modified version of [GP3]. 
For surfaces with Kodaira dimension 0 we show precisely the following:

Theorem 0.2. Let $X$ be a minimal surface with Kodaira dimension 0 and let $B_{1}, \ldots, B_{n}$ be numerically equivalent, ample and base-point-free line bundles. Assume that the sectional genus of $B_{i}$ is greater than or equal to 4 if $X$ is an Enriques surface and greater than or equal to 3 if $X$ is a $\mathrm{K} 3$ surface. Then $B_{1} \otimes \cdots \otimes B_{n}$ satisfies $N_{p}$ for all $n \geqq p+1$ and $p \geqq 1$.

The proof of Theorem 0.2 and stronger versions of the theorem can be found for Enriques surfaces in Section 2, for Abelian and bielliptic surfaces in Section 4 and for K 3 surfaces in [GP 3]. In the case of K 3 surfaces we prove a stronger version of 0.1 imposing extra conditions on $B_{i}$ (see [GP3]). As a consequence of Theorem 0.2 we obtain the following:

Theorem 0.3. Let $X$ be a minimal surface with Kodaira dimension 0 , let $B$ be an ample and base-point-free line bundle, and let $A$ be an ample line bundle. If $n \geqq p+1$ and $p \geqq 1$, then the bundle $K_{X} \otimes B^{\otimes n}$ satisfies property $N_{p}$ and if $m \geqq 2 p+2$ and $p \geqq 1$, then the bundle $K_{X} \otimes A^{\otimes m}$ satisfies property $N_{p}$.

Theorem 0.3 recovers Kempf's result for Abelian surfaces and implies the already mentioned result (1) regarding Mukai's conjecture.

For surfaces of positive Kodaira dimension we prove results in the spirit of 0.1 for adjoint linear series and for powers of ample and base-point-free line bundles (see Theorems 5.1, 5.8 and 5.14). We apply these results to obtain the above mentioned results (3) and (5) regarding effective bounds for projective normality, normal presentation, and property $N_{p}$, and (4) on pluricanonical models of surfaces of general type (see Theorem 5.12 and Theorem 5.16). Our results on projective normality, normal presentation and higher syzygies of pluricanonical models recover and strengthen results of Ciliberto on projective normality. In particular, we show the following, which answers a question posed by Bombieri (in [Bo]):

Let $X$ be a surface of general type such that $p_{g} \geqq 2$ or $K_{X}^{2} \geqq 5$. If $n \geqq 5$, then the image of $X$ by $\left|K_{X}^{\otimes n}\right|$ is projectively normal.

Moreover we improve results of [Ci] in the case of regular surfaces (Corollary 5.6).

In other works, we apply the techniques developed in this article to study syzygies of higher dimensional varieties. We show results in the spirit of 0.1 for Fano varieties in [GP3] and for Calabi-Yau threefolds. In [GP4] we prove optimal results on very ampleness, projective normality and higher syzygies for Calabi-Yau threefolds. These results are similar in spirit to the well known results of St. Donat for K 3 surfaces and Lefschetz for Abelian varieties.

Another very interesting problem in this area is the relation between normal presentation and the Koszul property of coordinate rings. We show that whenever a line bundle on the variety under consideration (in this article) is normally presented then it embeds the variety with a Koszul homogeneous coordinate ring. This gives further evidence to the following (to paraphrase Arnold): Any homogeneous coordinate ring which has a serious reason for being quadratically presented is Koszul. In Section 3 we develop the necessary tools to tackle this problem and restrict ourselves to Enriques surfaces. In the subsequent sections we apply these tools to prove the result for other surfaces. 
A basic obstacle one encounters in the kind of problems we have been talking about in the previous paragraphs is the scarcity of techniques to compute Koszul cohomology groups of surfaces and higher dimensional varieties - as Green put it, there are more reasons to compute them than ways of computing them. In our experience, this is especially so if the adjoint linear series involves base-point-free or ample line bundles, to mention the case of Mukai's conjecture. In this article and in previous ones we have developed techniques to compute these cohomology groups. Firstly in the proofs of the vanishings leading to results on higher syzygies we use induction on the number of ample and basepoint-free line bundles composing the line bundle we are studying. To prove the vanishings which correspond to the first step of the induction we have found it necessary to use the intrinsic geometric properties of the varieties under consideration. We make here a distinction between two classes of varieties: those with irregularity $h^{1}\left(\mathcal{O}_{X}\right)>0$ and those with irregularity $h^{1}\left(\mathcal{O}_{X}\right)=0$. In the former case we use arguments involving CastelnuovoMumford regularity and the existence of enough homologically trivial line bundles to show the surjectivity of certain multiplication maps of vector bundles on the variety. In the latter case (comprising among other $\mathrm{K} 3$ surfaces, Fano varieties, Enriques surfaces, anticanonical rational surfaces and Calabi-Yau threefolds) we give uniform proofs using induction on the dimension of the variety. Precisely we choose a suitable divisor on the variety (we point out to the reader that it is not a hyperplane section; such a choice would lead us to line bundles of low degree on curves, which would be of little use for us). Then we restrict the vector bundles on the surface arising in the context of Koszul cohomology to the divisor, construct a filtration of the restricted bundle (which happens to be the HarderNarasimhan filtration) and play the game in lower dimension. Doing this, we reduce the question of the surjectivity of multiplication maps on the ambient variety to a question of surjectivity of multiplication maps on the divisor. This allows us to use eventually results on surjectivity of multiplication maps of vector bundles on curves, like the technical (and beautiful) results by Butler and Pareschi, [Bu], Proposition 2.2 and [P2], Corollary 4.

The article is organized as follows. In the first section we introduce the Koszul cohomology basics used in the article and Castelnuovo-Mumford regularity in connection with surjectivity of multiplication maps of vector bundles. The goal of this section is to prove a general result, Theorem 1.3, which demonstrates how far we can go using a general technique like Castelnuovo-Mumford regularity. In fact, this theorem becomes our start line in the quest we pursue throughout the rest of the paper to obtain more precise, sharper results which cannot be proved by means of these general arguments.

Section 2 deals with very ampleness, projective normality, and higher syzygies on Enriques surfaces. Here we introduce the methods we develop to work with surfaces with irregularity 0 . In subsequent sections similar arguments are dealt with in a less detailed way.

Section 3 is devoted to the study of the Koszul property on Enriques surfaces. A great amount of machinery is developed which is later used in the remaining sections when studying the same problem on other surfaces.

Section 4 deals with Abelian and bielliptic surfaces, completing the picture of surfaces with Kodaira dimension 0.

Section 5 deals with surfaces of positive Kodaira dimension. Among other things we carry out the study of pluricanonical series on surfaces of general type. 
Finally, in the appendix, we show that our main results go through for singular surfaces, obtaining in Kodaira dimension 0 the same bounds towards Mukai's conjecture that we obtain for smooth surfaces.

Convention. Throughout this article we work over an algebraically closed field of characteristic 0. Except in the appendix, for us surface will always mean minimal and smooth algebraic surface. We will denote numerical equivalence of divisors by $\equiv$.

Definition. Let $X$ be a projective variety and let $L$ be a very ample line bundle on $X$. We say that $L$ is normally generated or that satisfies the property $N_{0}$, if $|L|$ embeds $X$ as a projectively normal variety. We say that $L$ is normally presented or that $L$ satisfies the property $N_{1}$ if $L$ satisfies property $N_{0}$ and, in addition, the homogeneous ideal of the image of $X$ by $|L|$ is generated by quadratic equations. We say that $L$ satisfies the property $N_{p}$ for $p>1$, if $L$ satisfies property $N_{1}$ and the free resolution of the homogeneous ideal of $X$ embedded by $|L|$ is linear until the $p$ th-stage.

\section{A general result on syzygies of algebraic varieties}

As we mentioned in the introduction, Green interpreted the Betti numbers of the minimal free resolution of the coordinate ring of an embedded projective variety in terms of Koszul cohomology. Concretely, let $X$ be a projective variety, and let $F$ be a globally generated vector bundle on $X$. We define the bundle $M_{F}$ as follows:

$$
0 \rightarrow M_{F} \rightarrow H^{0}(F) \otimes \mathcal{O}_{X} \rightarrow F \rightarrow 0 .
$$

If $L$ is an ample line bundle on $X$ and all its positive powers are nonspecial one has the following characterization of the property $N_{p}$ :

Theorem 1.2. Let $L$ be an ample, globally generated line bundle on a variety $X$. If the group $H^{1}\left(\wedge^{p^{\prime}+1} M_{L} \otimes L^{\otimes s}\right)$ vanishes for all $0 \leqq p^{\prime} \leqq p$ and all $s \geqq 1$, then $L$ satisfies the property $N_{p}$. If in addition $H^{1}\left(L^{\otimes r}\right)=0$, for all $r \geqq 1$, then the above is a necessary and sufficient condition for $L$ to satisfy property $N_{p}$.

We will obtain our results on syzygies using the previous lemma. For the proof of it we refer to [EL], Section 1. Recall that we are working over an algebraically closed field of characteristic 0 , thus in our proofs we will check the vanishings of $H^{1}\left(M_{L}^{\otimes p^{\prime}+1} \otimes L^{\otimes s}\right)$ rather than see directly the vanishings of $H^{1}\left(\wedge^{p^{\prime}+1} M_{L} \otimes L^{\otimes s}\right)$.

The purpose of this section is to prove a general result about Koszul cohomology and, by the above lemma, about syzygies of varieties of arbitrary dimension.

Theorem 1.3. Let $X$ be a projective variety. Let $B$ be a base-point-free line bundle on $X$ with regularity $r$. If $n \geqq \max (r+p-2, p), p \geqq 1$ and $m \geqq \max (r, 1)$, then

$$
H^{i}\left(M_{B^{8 m}}^{\otimes p+1} \otimes B^{\otimes n+2-i}\right)=0 \quad \text { for all } i \geqq 1 .
$$

In particular, $H^{1}\left(M_{B^{\otimes m}}^{\otimes p+1} \otimes B^{\otimes n+1}\right)=0$ and if $B$ is ample and $n \geqq \max (r+p-2, r, p)$, then $B^{\otimes n+1}$ satisfies the property $N_{p}$. 
To prove the theorem we will need the following

Lemma 1.4. Let $X$ and $B$ be as in Theorem 1.3. If $n \geqq r-1$ and $m \geqq 1$, then

$$
H^{1}\left(M_{B^{\otimes m}} \otimes B^{\otimes n+1}\right)=0 .
$$

In particular, if $B$ is ample, then $B^{\otimes n+1}$ satisfies the property $N_{0}$.

Proof. Since $n+1 \geqq r, H^{1}\left(B^{\otimes n+1}\right)=0$. Thus, tensoring the sequence (1.1) relative to $B^{\otimes m}$ with $B^{\otimes n+1}$ and taking global sections one sees that it is enough to check that the multiplication map

$$
H^{0}\left(B^{\otimes m}\right) \otimes H^{0}\left(B^{\otimes n+1}\right) \rightarrow H^{0}\left(B^{\otimes m+n+1}\right)
$$

surjects. To see that, we use the following useful observation:

Observation 1.4.1. Let $E$ and $L_{1}, \ldots, L_{r}$ be coherent sheaves on a variety $X$. Consider the map $H^{0}(E) \otimes H^{0}\left(L_{1} \otimes \cdots \otimes L_{r}\right) \stackrel{\psi}{\longrightarrow} H^{0}\left(E \otimes L_{1} \otimes \cdots \otimes L_{r}\right)$ and the maps

$$
\begin{gathered}
H^{0}(E) \otimes H^{0}\left(L_{1}\right) \stackrel{\alpha_{1}}{\longrightarrow} H^{0}\left(E \otimes L_{1}\right), \\
H^{0}\left(E \otimes L_{1}\right) \otimes H^{0}\left(L_{2}\right) \stackrel{\alpha_{2}}{\longrightarrow} H^{0}\left(E \otimes L_{1} \otimes L_{2}\right), \\
\cdots, \\
H^{0}\left(E \otimes L_{1} \otimes \cdots \otimes L_{r-1}\right) \otimes H^{0}\left(L_{r}\right) \stackrel{\alpha_{r}}{\longrightarrow} H^{0}\left(E \otimes L_{1} \otimes \cdots \otimes L_{r}\right) .
\end{gathered}
$$

If $\alpha_{1}, \ldots, \alpha_{r}$ are surjective then $\psi$ is also surjective.

In our case, we set $L_{i}=B$ and $E=B^{\otimes n+1}$, and to see that the maps $\alpha_{i}$ are surjective we use the following generalization by Mumford of a lemma of Castelnuovo (see [Mu]; note that the assumption of ampleness is unnecessary):

(1.4.2) Let $L$ be a base-point-free line bundle on a variety $X$ and let $\mathscr{F}$ be a coherent sheaf on $X$. If $H^{i}\left(\mathscr{F} \otimes L^{-1}\right)=0$ for all $i \geqq 1$, then the multiplication map

$$
H^{0}\left(\mathscr{F} \otimes L^{\otimes i}\right) \otimes H^{0}(L) \rightarrow H^{0}\left(\mathscr{F} \otimes L^{\otimes i+1}\right)
$$

is surjective for all $i \geqq 0$.

Finally, the vanishings required according to (1.4.2) follow from our assumption on regularity.

(1.5) Proof of Theorem 1.3. The proof is by induction on $p$. We prove the result for $p=1$. First we show that

$$
H^{1}\left(M_{B^{8 m}}^{\otimes 2} \otimes B^{\otimes n+1}\right)=0 \quad \text { for all } m \geqq r, 1 \text { and all } n \geqq r-1,1 .
$$


We will use (1.4.2) and Observation 1.4.1 to prove this statement. Observe that tensoring the sequence (1.1) with $M_{B^{\otimes_{m}}} \otimes B^{\otimes n+1}$ and taking global sections yields the following long exact sequence:

$$
\begin{gathered}
H^{0}\left(M_{B^{\otimes m}} \otimes B^{\otimes n+1}\right) \otimes H^{0}\left(B^{\otimes m}\right) \stackrel{\gamma}{\longrightarrow} H^{0}\left(M_{B^{\otimes m}} \otimes B^{\otimes m+n+1}\right) \\
\rightarrow H^{1}\left(M_{B^{\otimes m}}^{\otimes 2} \otimes B^{\otimes n+1}\right) \rightarrow H^{1}\left(M_{B^{\otimes m}} \otimes B^{\otimes n+1}\right) \otimes H^{0}\left(B^{\otimes m}\right) .
\end{gathered}
$$

The last term in the above sequence is zero by Lemma 1.4. Thus it is enough to prove that $\gamma$ surjects. By Observation 1.4.1 it is enough to show that the multiplication map

$$
H^{0}\left(M_{B^{\otimes m}} \otimes B^{\otimes n+1}\right) \otimes H^{0}(B) \rightarrow H^{0}\left(M_{B^{\otimes m}} \otimes B^{\otimes n+2}\right)
$$

surjects for all $m \geqq r, 1$ and all $n \geqq r-1,1$. Since $B$ is base-point-free, by (1.4.2) we need to check the vanishings $H^{i}\left(M_{B^{\otimes m}} \otimes B^{\otimes n+1-i}\right)=0$ for all $i \geqq 1, m \geqq r, 1$ and $n \geqq r-1,1$. For $i \geqq 2$, we tensor the sequence (1.1) corresponding to $B^{\otimes m}$ with $B^{\otimes_{n+1-i}}$ and take global sections. The vanishings then follow from our assumption on the regularity of $B$. Since $m \geqq r$ and $n \geqq r-1$ it follows in particular that $H^{1}\left(B^{\otimes m}\right)=H^{1}\left(B^{\otimes n}\right)=0$, hence the vanishing required for $i=1$ is equivalent to the vanishing of $H^{1}\left(M_{B^{\otimes n}} \otimes B^{\otimes m}\right)$, which follows in turn from Lemma 1.4.

The vanishings of $H^{i}\left(M_{B^{\otimes m}}^{\otimes 2} \otimes B^{\otimes n+2+i}\right)$ for all $m \geqq 1$, all $i \geqq 2$ and all $n \geqq r-1$ follow from (1.1), Lemma 1.4, and the assumption on regularity.

Let us now assume that the desired vanishings occur for $p-1$. We therefore have:

$$
\begin{gathered}
H^{i}\left(M_{B^{\otimes m}}^{\otimes p} \otimes B^{\otimes n+2-i}\right)=0 \quad \text { for all } n \geqq \max (p+r-3, p-1), \\
\text { all } m \geqq \max (r, 1) \text { and all } i \geqq 1 .
\end{gathered}
$$

We first prove the desired vanishing for $p$ and $i=1$. By tensoring the sequence (1.1) with $M_{B^{\otimes m}}^{\otimes p} \otimes B^{\otimes n+1}$ and taking global sections one sees that the desired vanishing can be obtained by showing the surjectivity of the multiplication map $\delta$ sitting in the long exact sequence

$$
\begin{aligned}
& H^{0}\left(M_{B^{\otimes m}}^{\otimes p} \otimes B^{\otimes n+1}\right) \otimes H^{0}\left(B^{\otimes m}\right) \stackrel{\delta}{\longrightarrow} H^{0}\left(M_{B^{\otimes m}}^{\otimes p} \otimes B^{\otimes m+n+1}\right) \\
& \rightarrow H^{1}\left(M_{B^{\otimes m}}^{\otimes p+1} \otimes B^{\otimes n+1}\right) \rightarrow H^{1}\left(M_{B^{\otimes m}}^{\otimes p} \otimes B^{\otimes n+1}\right) \otimes H^{0}\left(B^{\otimes m}\right) .
\end{aligned}
$$

The last term is zero by induction assumption. In order to prove the surjectivity of $\delta$ we use Observation 1.4.1. By Observation 1.4.1 it suffices to show the surjectivity of the map

$$
H^{0}\left(M_{B^{\otimes m}}^{\otimes p} \otimes B^{\otimes n+1}\right) \otimes H^{0}(B) \stackrel{\varepsilon}{\longrightarrow} H^{0}\left(M_{B^{\otimes m}}^{\otimes p} \otimes B^{\otimes n+2}\right)
$$

for all $n \geqq p+r-2, p$ and all $m \geqq r, 1$. To prove the surjectivity of $\varepsilon$ we use (1.4.2). According to it, it suffices that the groups $H^{i}\left(M_{B^{\otimes m}}^{\otimes p} \otimes B^{\otimes n+1-i}\right)$ vanish, which follows by induction. 
Finally, to show that $H^{i}\left(M_{B^{\otimes m}}^{\otimes p+1} \otimes B^{\otimes n+2-i}\right)=0$, for all $i \geqq 2$ we consider again sequence (1.1) associated to $B^{\otimes m}$, tensor it with $M_{B^{\otimes m}}^{\otimes p} \otimes B^{\otimes n+2-i}$ and take global sections. Then the vanishings follow again from induction hypothesis.

The fact that $B^{\otimes n+1}$ satisfies the property $N_{p}$ follows from the vanishing of $H^{1}\left(M_{B^{\otimes n+1}}^{\otimes p^{\prime}} \otimes B^{\otimes s(n+1)}\right)$ for all $1 \leqq p^{\prime} \leqq p$ and all $s \geqq 1$, from Lemma 1.4 and from Theorem 1.2 .

The theorem just proven, which might seem at first glance somehow vague, holds however the power to unify several results for different kinds of varieties: it yields information about pluricanonical embeddings of surfaces of general type (Corollary 5.11). It also implies the following corollary concerning varieties of arbitrary dimension and canonical divisor numerically trivial, an example of which are Calabi-Yau $n$-folds:

Corollary 1.6. Let $X$ be a smooth variety of dimension $m$ with $K_{X} \equiv 0$ and let $B$ be ample and a base-point-free line bundle. Let $L=B^{\otimes n+1}$. If $n \geqq p+m-1$, then $L$ satisfies property $N_{p}$. In particular, if $X$ is a Calabi-Yau threefold, $B$ is an ample and base-point-free line bundle on $X, n \geqq p+3$ and $p \geqq 1$, then $B^{\otimes n}$ satisfies property $N_{p}$.

Proof. The result is a straightforward consequence of Theorem 1.3, since by Kodaira vanishing Theorem, $B$ is $(n+1)$-regular.

Theorem 1.3 also implies a result for surfaces with $p_{g}=0$ (among them elliptic ruled surfaces, Enriques surfaces and bielliptic surfaces):

Theorem 2.2 ([GP2]). Let $X$ be a surface with $p_{g}=0$. Let $B$ be a nonspecial, ample, and base-point-free line bundle. Then $B^{\otimes p+1}$ satisfies the property $N_{p}$ for all $p \geqq 1$.

Therefore Theorem 1.3 and its corollaries are a good starting point for our study of syzygies of varieties. However, if one focuses on the particular examples and uses the specific geometry of the varieties in question, one can expect to obtain sharper and more complete results. Precisely this was done for elliptic ruled surfaces in [GP2] and is done for Enriques surfaces in Section 2, for bielliptic surfaces in Section 4, for surfaces of general type in Section 5.

\section{Syzygies of Enriques surfaces}

In Section 1 we proved a general theorem, Theorem 1.3, which unifies a number of results for different kinds of varieties. In this section we focus on Enriques surfaces. The geometric genus of an Enriques surface is 0 and, in characteristic 0, a globally generated line bundle over an Enriques surface has null higher cohomology, hence it is 2-regular. Therefore the starting point of our study of syzygies of Enriques surfaces is the following theorem, corollary of Theorem 1.3, which fits indeed in (0.1):

Theorem 2.1 (cf. [GP2], Corollary 2.7.1). Let $X$ be an Enriques surface. Let $B$ be a base-point-free line bundle. Then the image of $X$ by $\left|B^{\otimes p+1}\right|$ satisfies property $N_{p}$, for all $p \geqq 1$. If in addition $B$ is ample then $B^{\otimes p+1}$ is very ample and satisfies the property $N_{p}$, for all $p \geqq 1$. 
Our intention now is to study a more general class of line bundles (namely, tensor products of $p+1$ different base-point-free line bundles), and in particular, adjoint line bundles. For that we need to follow a different approach: roughly, we are going to use "induction on the dimension", in the sense explained in the introduction. This approach will unfold throughout this section and the machinery developed along the way will be used for other results of this article, concretely in Sections 3 and 5. We now resume with a result about normal generation:

Theorem 2.2. Let $X$ be an Enriques surface. Let $B_{1}, B_{1}^{\prime}, B_{2}$ and $B_{2}^{\prime}$ be ample and base-point-free line bundles on $X$, such that $B_{1} \equiv B_{1}^{\prime}, B_{2} \equiv B_{2}^{\prime}$ and, either $B_{1} \cdot B_{2} \geqq 4, B_{1}^{2} \geqq 6$, and $B_{2}^{2} \geqq 6$ or $B_{1} \cdot B_{2} \geqq 5$. Let $L=B_{1}^{\otimes r} \otimes B_{2}^{\otimes s}$ and $L^{\prime}=B_{1}^{\prime \otimes k} \otimes B_{2}^{\prime \otimes l}$. If $r, s, k \geqq 1$, and $l \geqq 0$, then the map $H^{0}(L) \otimes H^{0}\left(L^{\prime}\right) \stackrel{\alpha}{\longrightarrow} H^{0}\left(L \otimes L^{\prime}\right)$ surjects and

$$
H^{1}\left(M_{L} \otimes L^{\prime}\right)=H^{1}\left(M_{L}^{\prime} \otimes L\right)=0
$$

In particular, $L$ is very ample and satisfies property $N_{0}$.

Before we go on with the proof of Theorem 2.2, we isolate for convenience three ingredients of the argument, which will be used in many other instances. The first is an observation on the relation between the surjectivity of multiplication maps, and the surjectivity of its restrictions to divisors. The other two are a result do Butler and another one due to Pareschi, about the surjectivity of multiplication maps of vector bundles on curves.

Observation 2.3. Let $X$ be a regular variety (i.e., a variety such that $H^{1}\left(\mathcal{O}_{X}\right)=0$ ). Let $E$ be a vector bundle on $X$, let $C$ be a divisor such that $L=\mathcal{O}_{X}(C)$ is a globally generated line bundle and $H^{1}\left(E \otimes L^{-1}\right)=0$. If the multiplication map

$$
H^{0}\left(E \otimes \mathcal{O}_{C}\right) \otimes H^{0}\left(L \otimes \mathcal{O}_{C}\right) \rightarrow H^{0}\left(E \otimes L \otimes \mathcal{O}_{C}\right)
$$

surjects, then the map $H^{0}(E) \otimes H^{0}(L) \rightarrow H^{0}(E \otimes L)$ also surjects.

Proof. We construct the following commutative diagram:

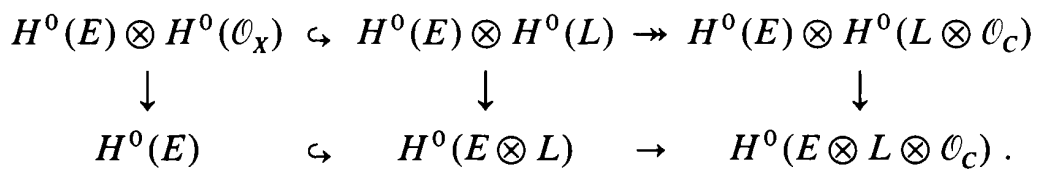

The surjectivity of the left hand side vertical map is obvious. The surjectivity of the right hand side vertical map follows by hypothesis. The exactness of the top horizontal sequence follows from the fact that $X$ is regular. The claim is the surjectivity of the middle vertical map.

Proposition $2.4([\mathrm{Bu}]$, Proposition 2.2). Let $E$ and $F$ be semistable vector bundles over a curve $C$ such that $E$ is generated by its global sections. If 
(1) $\mu(F)>2 g$, and

(2) $\mu(F)>2 g+\operatorname{rank}(E)(2 g-\mu(E))-2 h^{1}(E)$,

then the multiplication map $H^{0}(E) \otimes H^{0}(F) \rightarrow H^{0}(E \otimes F)$ surjects.

Proposition 2.5 ([P2], Corollary 4; see also [EKS], Theorem 2). Let $N$ and $L$ be two base-point-free line bundles on $C$ such that:

(a) at least one of them is very ample;

(b) $h^{0}(N), h^{0}(L) \geqq 3$ and

(c) $\operatorname{deg} N+\operatorname{deg} L \geqq \max \left(3 g-3,4 g+1-2 h^{1}(N)-2 h^{1}(L)-\operatorname{Cliff}(C)\right)$.

Then the multiplication map

$$
H^{0}(L) \otimes H^{0}(N) \rightarrow H^{0}(L \otimes N)
$$

is surjective.

(2.6) Proof of Theorem 2.2. Note first that, since we are working over a field of characteristic 0 , any base-point-free line bundle on $X$ has null higher cohomology. If we twist the sequences (1.1) relative to $L$ and $L^{\prime}$ by $L^{\prime}$ and $L$ respectively and take global sections, we see at once that $H^{1}\left(M_{L} \otimes L^{\prime}\right)=H^{1}\left(M_{L}^{\prime} \otimes L\right)$ and equal to the cokernel of

$$
H^{0}(L) \otimes H^{0}\left(L^{\prime}\right) \stackrel{\alpha}{\longrightarrow} H^{0}\left(L \otimes L^{\prime}\right)
$$

To see that $\alpha$ indeed surjects, we use Observation 1.4.1. According to it we want to check that several (possibly more than one) multiplication maps surject. We check here the first one; the surjectivity of the rest can be seen in the same way. The map in question is

$$
H^{0}(L) \otimes H^{0}\left(B_{1}^{\prime}\right) \stackrel{\beta}{\longrightarrow} H^{0}\left(L \otimes B_{1}^{\prime}\right)
$$

To see the surjectivity of $\beta$, we consider a smooth irreducible curve $C$ in $\left|B_{1}^{\prime}\right|$ (such curve exists by Bertini's Theorem because $B_{1}^{\prime}$ is ample and base-point-free) and use Observation 2.3. It is therefore enough to check that

$$
H^{0}\left(L \otimes \mathcal{O}_{C}\right) \otimes H^{0}\left(B_{1}^{\prime} \otimes \mathcal{O}_{C}\right) \stackrel{\gamma}{\longrightarrow} H^{0}\left(L \otimes B_{1}^{\prime} \otimes \mathcal{O}_{C}\right)
$$

surjects. For that, if $B_{1} \cdot B_{2} \geqq 5$, we may apply Proposition 2.4 . Indeed, the line bundle $B_{1}^{\prime}$ is globally generated, and by adjunction

$$
\mu\left(L \otimes \mathcal{O}_{C}\right)=\operatorname{deg} L \otimes \mathcal{O}_{C} \geqq 2 g(C)+3>2 g(C)+2 .
$$

If $B_{1} \cdot B_{2}=4$ and $B_{1}^{2} \geqq 6$, then $g(C) \geqq 4$ and, since $C$ is irreducible, it follows that it is non-hyperelliptic (cf. [CD], Proposition 4.5.1). Then the surjectivity of $\gamma$ follows from Proposition 2.5. 
As a corollary of Theorem 2.2 we prove a stronger version of the conjecture of Mukai, in the case of Enriques surfaces and for the property $N_{0}$. To see that we use the following

Lemma 2.7. Let $A_{1}$ and $A_{2}$ be two ample divisors on a surface $X$ with Kodaira dimension 0. Then $A_{1} \otimes A_{2}$ is base-point-free.

Proof. Since $K_{X} \equiv 0,\left(A_{1} \otimes A_{2}\right)^{2} \geqq 5$. By hypothesis $A_{1} \otimes A_{2}$ is ample. If $A_{1} \otimes A_{2}$ were not base-point-free, it would follow from Reider's theorem that there would exist an effective divisor $E$ such that one of the following holds:

(a) $\left(A_{1} \otimes A_{2}\right) \cdot E=0$ and $E^{2}=-1$

or

(b) $\left(A_{1} \otimes A_{2}\right) \cdot E=1$ and $E^{2}=0$.

None of the two possibilities can occur since, $A_{i}$ being ample, $A_{i} \cdot E \geqq 1$.

Corollary 2.8. Let $X$ be an Enriques surface and $A_{1}, \ldots, A_{n}$ ample line bundles on $X$. Let $L=K_{X} \otimes A_{1} \otimes \cdots \otimes A_{n}$. If $n \geqq 4$, then $L$ satisfies property $N_{0}$.

Proof. By Lemma 2.7, $K_{X} \otimes A_{1} \otimes A_{2}$ and $A_{3} \otimes \cdots \otimes A_{n}$ are base-point-free line bundles. There are furthermore ample, and, by adjunction,

$$
\left(K \otimes A_{1} \otimes A_{2}\right)^{2} \geqq 6, \quad\left(A_{3} \otimes \cdots \otimes A_{n}\right)^{2} \geqq 6 \quad \text { and } \quad\left(K \otimes A_{1} \otimes A_{2}\right) \cdot\left(A_{3} \otimes \cdots \otimes A_{n}\right) \geqq 4
$$

Then the result follows from Theorem 2.2.

We now generalize these results to higher syzygies. To do so, we need another two lemmas. In the case in which $q$ is a curve $C$, the former allows us to pass from a multiplication map involving non-semistable bundles (note that $M_{F} \otimes \mathcal{O}_{C}$ is unstable if

$$
\left.H^{1}(L \otimes \mathcal{O}(-C))=0\right)
$$

to a multiplication map involving semistable bundles. This situation is of course easier to handle. The latter lemma deals with positivity and semistability of bundles on curves. They will not only be used for the arguments in the remaining of this section but also in Section 3 and 5 .

Lemma 2.9. Let $X$ be a projective variety, let $q$ be a nonnegative integer and let $F_{i}$ be a base-point-free line bundle on $X$ for all $1 \leqq i \leqq q$. Let $Q$ be an effective line bundle on $X$ and let $\mathfrak{q}$ be a reduced and irreducible member of $|Q|$. Let $R$ be a line bundle and $G$ a sheaf on $X$ such that 
1. $H^{1}\left(F_{i} \otimes Q^{*}\right)=0$,

2. $H^{0}\left(M_{\left(F_{i} \otimes \mathcal{O}_{q}\right)} \otimes \cdots \otimes M_{\left(F_{q^{\prime}} \otimes \mathcal{O} q\right)} \otimes R \otimes \mathcal{O}_{\mathfrak{q}}\right) \otimes H^{0}(G)$

$\rightarrow H^{0}\left(M_{\left(F_{i_{1}} \otimes \mathcal{O}_{q}\right)} \otimes \cdots \otimes M_{\left(F_{\left.i_{q^{\prime}} \otimes \mathcal{Q}\right)}\right.} \otimes R \otimes G \otimes \mathcal{O}_{\mathbf{q}}\right)$ surjects for all $0 \leqq q^{\prime} \leqq q$.

Then, for all $0 \leqq q^{\prime \prime} \leqq q$ and any subset $\left\{j_{k}\right\} \leqq\{i\}$ with \#\{$\left.j_{k}\right\}=q^{\prime \prime}$ and for all $0 \leqq k^{\prime} \leqq q^{\prime \prime}$,

$$
\begin{aligned}
& H^{0}\left(M_{F_{j_{1}}} \otimes \cdots \otimes M_{F_{j k^{\prime}}} \otimes M_{\left(F_{j_{k^{\prime}}+1} \otimes \mathcal{O} q\right)} \otimes \cdots \otimes M_{\left(F_{j_{q^{\prime}}} \otimes \mathcal{O} q\right)} \otimes R \otimes \mathcal{O}_{\mathrm{q}}\right) \otimes H^{0}(G) \\
& \rightarrow H^{0}\left(M_{F_{j_{1}}} \otimes \cdots \otimes M_{F_{j_{k^{\prime}}}} \otimes M_{\left(F_{j_{k^{\prime}+1}} \otimes \mathcal{O} q\right)} \otimes \cdots \otimes M_{\left(F_{j^{\prime}}{ }^{\prime \prime} \otimes \mathcal{O} q\right)} \otimes G \otimes R \otimes \mathcal{O}_{q}\right)
\end{aligned}
$$

surjects.

Proof. We prove the result by induction on $q^{\prime \prime}$. For $q^{\prime \prime}=0$ the corresponding statement is just Condition 2 when $q=0$. Assume that the result is true for $q^{\prime \prime}-1$. In order to prove the result for $q^{\prime \prime}$ we will use induction on $k^{\prime}$. If $k^{\prime}=0$, the statement is again just Condition 2. Assume that the result is true for $k^{\prime}-1$. Now for any $F$ globally generated vector bundle and for any effective divisor $\mathfrak{q}$ such that $H^{1}\left(F \otimes Q^{*}\right)=0$, for $Q=\mathcal{O}(\mathfrak{q})$, we have this commutative diagram:

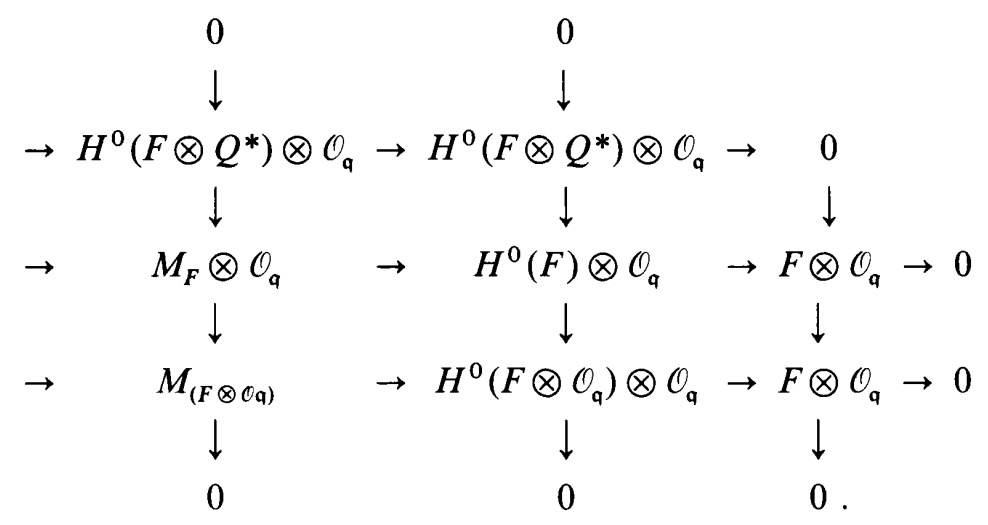

We are interested in the left hand side vertical exact sequence:

$$
0 \rightarrow H^{0}\left(F \otimes Q^{*}\right) \otimes \mathcal{O}_{\mathfrak{q}} \rightarrow M_{F} \otimes \mathcal{O}_{\mathfrak{q}} \rightarrow M_{\left(F \otimes \mathcal{O}_{\mathfrak{q}}\right)} \rightarrow 0
$$

By Condition 1, $F$ can be taken to be $F_{j_{k^{\prime}}}$ Tensoring (2.9.1) by

$$
M_{F_{j_{1}}} \otimes \cdots \otimes M_{F_{j_{k^{\prime}-1}}} \otimes M_{\left(F_{j_{k^{\prime}}+1} \otimes \mathcal{O} \mathfrak{q}\right)} \otimes \cdots \otimes M_{\left(F_{j_{q^{\prime \prime}}} \otimes \mathcal{G}\right)} \otimes R \otimes \mathcal{O}_{\mathfrak{q}}
$$

taking global sections and tensoring by $H^{0}(G)$ we obtain this commutative diagram:

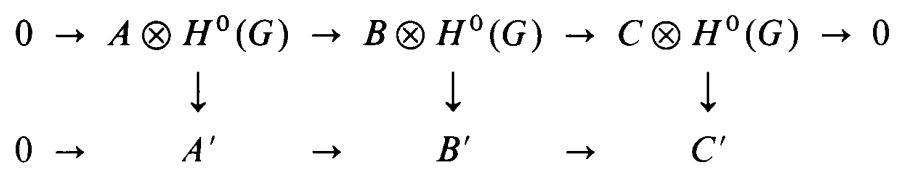

11 Journal für Mathematik. Band 506 
where

and

$$
\begin{aligned}
& A=H^{0}\left(F_{j_{k^{\prime}}} \otimes Q^{*}\right) \otimes H^{0}\left(\bigotimes_{r=1}^{k^{\prime}-1} M_{F_{j_{r}}} \otimes \bigotimes_{r=k^{\prime}+1}^{q^{\prime \prime}} M_{\left(F_{j_{r}} \otimes \mathcal{O} q\right)} \otimes R \otimes \mathcal{O}_{q}\right), \\
& B=H^{0}\left(\bigotimes_{r=1}^{k^{\prime}} M_{F_{j_{r}}} \otimes \bigotimes_{r=k^{\prime}+1}^{q^{\prime \prime}} M_{\left(F_{j_{r}} \otimes \mathcal{O} q\right)} \otimes R \otimes \mathcal{O}_{\mathrm{q}}\right) \\
& C=H^{0}\left(\bigotimes_{r=1}^{k^{\prime}-1} M_{F_{j_{r}}} \otimes \bigotimes_{r=k^{\prime}}^{q^{\prime \prime}} M_{\left(F_{j_{r}} \otimes \mathcal{G}\right)} \otimes R \otimes \mathcal{O}_{q}\right),
\end{aligned}
$$

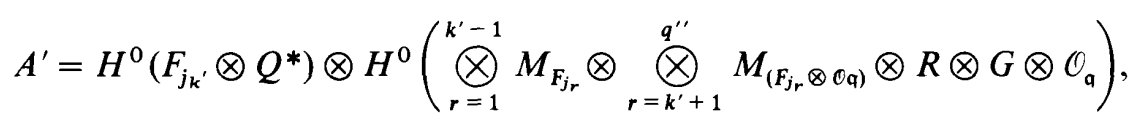

$$
\begin{aligned}
& B^{\prime}=H^{0}\left(\bigotimes_{r=1}^{k^{\prime}} M_{F_{j_{r}}} \otimes \bigotimes_{r=k^{\prime}+1}^{q^{\prime \prime}} M_{\left(F_{j_{r}} \otimes \mathcal{O} q\right)} \otimes R \otimes G \otimes \mathcal{O}_{q}\right)
\end{aligned}
$$

$$
C^{\prime}=H^{0}\left(\bigotimes_{r=1}^{k^{\prime}-1} M_{F_{j_{r}}} \otimes \bigotimes_{r=k^{\prime}}^{q^{\prime \prime}} M_{\left(F_{j_{r}} \otimes \mathcal{G}\right)} \otimes R \otimes G \otimes \mathcal{O}_{\mathfrak{q}}\right)
$$

The top horizontal exact sequence is certainly surjective: this follows from chasing the diagram after having taken cohomology. The left hand side vertical sequence surjects by the induction hypothesis on $q^{\prime \prime}$ and the right hand side exact sequence surjects by induction on $k^{\prime}$ (we have assumed the result to be true for $q^{\prime \prime}-1$ and $k^{\prime}-1$ ). Therefore we obtain the surjectivity of the vertical sequence sitting in the middle of the commutative diagram.

Lemma 2.10. Let $E$ be a semistable vector bundle with $\mu(E)>2 g$ and $F$ a vector bundle on a curve $C$ of genus $g$.

(1) If $\mu(F) \geqq 2 g+4$, then $\mu\left(M_{E} \otimes F\right)>2 g+2$.

(2) If $\mu(F) \geqq 2 g+2$, then $\mu\left(M_{E} \otimes F\right)>2 g$.

Moreover, if $F$ is in addition semistable, then $M_{E} \otimes F$ is semistable.

Proof. Since $E$ is semistable and $\mu(E)>2 g, E$ is globally generated and $h^{1}(E)=0$, hence the vector bundle $M_{E}$ is defined and has slope

$$
\mu\left(M_{E}\right)=\frac{-\mu(E)}{\mu(E)-g} .
$$

Then, for (1), $\mu\left(M_{E} \otimes F\right) \geqq \frac{-\mu(E)}{\mu(E)-g}+2 g+4$. Thus if $\frac{-\mu(E)}{\mu(E)-g}+2 g+4>2 g+2$ we are done, but that inequality is equivalent to $\mu(E)>2 g$. The proof of (2) is analogous. Now, if $F$ is semistable by [Bu], Theorem 1.12 and [Mi], Corollary $3.7, M_{E} \otimes F$ is also semistable. 
Theorem 2.11. Let $X$ be an Enriques surface. Let $B_{1}, B_{1}^{\prime}, B_{2}$ and $B_{2}^{\prime}$ be two ample and base-point-free divisors such that $B_{1} \equiv B_{1}^{\prime}, B_{2} \equiv B_{2}^{\prime}$ and $B_{1} \cdot B_{2} \geqq 6$. Let $L=B_{1}^{\otimes s} \otimes B_{2}^{\otimes r}$ and $L^{\prime}=B_{1}^{\prime \otimes k} \otimes B_{2}^{\prime \otimes l}$. If $k, l, r, s \geqq 1$, then $H^{1}\left(M_{L}^{\otimes 2} \otimes L^{\prime}\right)=0$. In particular, $L^{\prime}$ satisfies property $N_{1}$.

Proof. The cohomology group $H^{1}\left(M_{L}^{\otimes 2} \otimes L^{\prime}\right)=0$ sits in the long exact sequence

$$
\begin{gathered}
H^{0}(L) \otimes H^{0}\left(M_{L} \otimes L^{\prime}\right) \stackrel{\alpha}{\longrightarrow} H^{0}\left(M_{L} \otimes L \otimes L^{\prime}\right) \\
\rightarrow H^{1}\left(M_{L}^{\otimes 2} \otimes L^{\prime}\right) \rightarrow H^{0}(L) \otimes H^{1}\left(M_{L} \otimes L^{\prime}\right),
\end{gathered}
$$

obtained by tensoring (1.1) relative to $L$ with $M_{L} \otimes L^{\prime}$ and taking global sections. The last term is zero by Theorem 2.2, thus it is enough to prove that $\alpha$ is surjective. To show the surjectivity of $\alpha$ we use Observation 1.4.1. According to it we need to check the surjectivity of several maps. Here we will only show the surjectivity of the first of them, since the rest are analogous:

$$
H^{0}\left(B_{1}\right) \otimes H^{0}\left(M_{L} \otimes L^{\prime}\right) \stackrel{\beta}{\longrightarrow} H^{0}\left(M_{L} \otimes L^{\prime} \otimes B_{1}\right)
$$

Let $C$ be a smooth member of $\left|B_{1}\right|$. From Theorem 2.2 it follows that

$$
H^{1}\left(M_{L} \otimes L^{\prime} \otimes B_{1}^{*}\right)=0,
$$

therefore we may apply Observation 2.3 to reduce the question of surjectivity of $\beta$ to the surjectivity of the following multiplication map on $C$ :

$$
H^{0}\left(B_{1} \otimes \mathcal{O}_{C}\right) \otimes H^{0}\left(M_{L} \otimes L^{\prime} \otimes \mathcal{O}_{C}\right) \rightarrow H^{0}\left(M_{L} \otimes L^{\prime} \otimes B_{1} \otimes \mathcal{O}_{C}\right) .
$$

By Lemma 2.9 it is enough to check that the following multiplication maps on $C$ are surjective:

$$
\begin{gathered}
H^{0}\left(B_{1} \otimes \mathcal{O}_{C}\right) \otimes H^{0}\left(L^{\prime} \otimes \mathcal{O}_{C}\right) \rightarrow H^{0}\left(B_{1} \otimes L^{\prime} \otimes \mathcal{O}_{C}\right) \\
H^{0}\left(B_{1} \otimes \mathcal{O}_{C}\right) \otimes H^{0}\left(M_{L \otimes \mathcal{O}_{C}} \otimes L^{\prime} \otimes \mathcal{O}_{C}\right) \stackrel{\gamma}{\longrightarrow} H^{0}\left(M_{L \otimes \mathcal{O}_{C}} \otimes L^{\prime} \otimes B_{1} \otimes \mathcal{O}_{C}\right) .
\end{gathered}
$$

The surjectivity of the first map was already seen within the course of proving Theorem 2.2. For $\gamma$, we use Proposition 2.4. Since $\operatorname{deg}\left(L \otimes \mathcal{O}_{C}\right)$ and $\operatorname{deg}\left(L^{\prime} \otimes \mathcal{O}_{C}\right)$ are both greater than or equal to $2 g+4$, it follows from Lemma 2.10 that the bundle $M_{L \otimes \mathscr{O}_{C}} \otimes L^{\prime} \otimes \mathcal{O}_{C}$ is semistable with slope strictly bigger than $2 g+2$. Then it follows from Proposition 2.4 that $\gamma$ is surjective and we are done. Now, since $L^{\prime}$ is ample, it follows from the vanishing of $H^{1}\left(M_{L^{\prime}}^{\otimes 2} \otimes L^{\prime \otimes s}\right)$ for all $s \geqq 1$, Theorem 2.2 and Theorem 1.2 , that $L^{\prime}$ satisfies property $N_{1}$.

We obtain the following corollary, which proves Mukai's conjecture (and when considering powers of the same ample bundle, improves his bound), regarding property $N_{1}$ for Enriques surfaces.

Corollary 2.12. Let $X$ be an Enriques surface. Let $A, A_{1}, \ldots, A_{n}$ be ample line bundles. Then the line bundles $K_{X} \otimes A^{\otimes m}$ and $K_{X} \otimes A_{1} \otimes \cdots \otimes A_{n}$ satisfy property $N_{1}$ if $m \geqq 4$ and $n \geqq 5$ respectively. 
Proof. For the former case, let $B_{1}=K_{X} \otimes A^{\otimes 2}$ and $B_{2}=A^{\otimes m-2}$. For the latter, let $B_{1}=K_{X} \otimes A_{1} \otimes A_{2} \otimes A_{3}$ and $B_{2}=A_{4} \otimes \cdots \otimes A_{n}$. In both cases, $B_{1}$ and $B_{2}$ are ample, and base-point-free by Lemma 2.7 . Furthermore, $B_{1} \cdot B_{2} \geqq 6$, consequently the result follows from Theorem 2.11.

To finish this section we show a result for higher syzygies of adjoint bundles. Before that we state a useful lemma dealing with the numerical nature of the property of base-pointfreeness.

Lemma 2.13. Let $X$ be a surface with nonnegative Kodaira dimension and let $B$ be an ample and base-point-free line bundle such that $B^{2} \geqq 5$. If $B^{\prime} \equiv B$, then $K_{X} \otimes B^{\prime}$ is ample and base-point-free. In particular, if $\kappa(X)=0, B^{\prime}$ is ample and base-point-free for all $B^{\prime} \equiv B$.

Proof. The line bundle $B^{\prime}$ is ample because ampleness is a numerical condition and has self-intersection greater than or equal to 5 . If $K_{X} \otimes B^{\prime}$ has base points, by Reider's theorem there is an effective divisor $E$ such that:

(a) $B^{\prime} \cdot E=0$ and $E^{2}=-1$

or

(b) $B^{\prime} \cdot E=1$ and $E^{2}=0$.

The former cannot happen because $B^{\prime}$ is ample. We will also rule out (b). The divisor $E$ must be irreducible and reduced because $B^{\prime}$ is ample and $B^{\prime} \cdot E=1$. On the other hand, the arithmetic genus of $E$ is greater than or equal to 1 . Now $B \cdot E=B^{\prime} \cdot E=1$ so $h^{0}\left(B \otimes \mathcal{O}_{E}\right) \leqq 1$. Since $B$ is base-point-free, $E$ should be a smooth rational curve and this is a contradiction.

Theorem 2.14. Let $X$ be an Enriques surface. Let $B$ be an ample and base-point-free line bundle such that $B^{2} \geqq 6$ and let $N, N^{\prime}$ be line bundles numerically equivalent to 0 (i.e., they are either trivial or equal to $\left.K_{X}\right)$. Let $L=B^{\otimes p+1+l} \otimes N, L^{\prime}=B^{\otimes p+1+k} \otimes N^{\prime}$ for $p \geqq 1$. Then $H^{1}\left(M_{L}^{\otimes p+1} \otimes L^{\prime}\right)$ vanishes for all $k, l \geqq 0$. In particular $L$ satisfies property $N_{p}$.

Proof. Since $B^{2} \geqq 6$, by Lemma 2.13 the line bundle $B \otimes N$ is also ample and base-point-free. The proof is by induction. The result is true for $p=1$ by Theorem 2.11 . We assume now the result to be true for $p-1$. In particular we have $H^{1}\left(M_{L}^{\otimes p} \otimes L^{\prime}\right)=0$. Tensoring the sequence (1.1) with $M_{L}^{\otimes p} \otimes L^{\prime}$ and taking global sections yields therefore the following long exact sequence:

$$
H^{0}(L) \otimes H^{0}\left(M_{L}^{\otimes p} \otimes L^{\prime}\right) \stackrel{\alpha}{\longrightarrow} H^{0}\left(M_{L}^{\otimes p} \otimes L \otimes L^{\prime}\right) \rightarrow H^{1}\left(M_{L}^{\otimes p+1} \otimes L^{\prime}\right) \rightarrow 0,
$$

thus it is enough to prove that the multiplication map $\alpha$ is surjective. Then by Observation 1.4.1 it is enough to see the surjectivity of

$$
H^{0}\left(B^{\prime}\right) \otimes H^{0}\left(M_{L}^{\otimes p} \otimes B^{\otimes p+1+k} \otimes N^{\prime}\right) \stackrel{\beta}{\longrightarrow} H^{0}\left(M_{L}^{\otimes p} \otimes B^{\otimes p+1+k} \otimes B^{\prime} \otimes N^{\prime}\right),
$$


where $B^{\prime}$ is either $B$ or $B \otimes N$. Now to complete the proof one can argue in two ways. One of them is using (1.4.2). The path to follow is shown in the proof of Theorem 1.3 but we outline here the steps to be taken. The first cohomology vanishing required,

$$
H^{1}\left(M_{L}^{\otimes p} \otimes B^{\otimes p+1+k} \otimes N^{\prime} \otimes B^{\prime *}\right)
$$

follows directly by induction. For the second cohomology vanishing one may observe that, after iteratively chasing the cohomology sequence, it follows by induction, from Theorem 2.2 and Kodaira Vanishing Theorem. The other way to argue is as for the surjectivity of $\beta$ in the proof of Theorem 2.11: one uses and apply Lemma 2.9 to reduce the problem to checking the surjectivity of multiplication maps on a curve.

Finally since $L$ is ample, Theorem 1.2 implies that $L$ satisfies $N_{p}$.

Corollary 2.15. Let $X$ be an Enriques surface, let $A$ be an ample line bundle and $B$ an ample and base-point-free line bundle on $X$. If $m \geqq p+1$, then $K_{X} \otimes B^{\otimes m}$ satisfies property $N_{p}$. If $n \geqq 2 p+2$, then $K_{X} \otimes A^{\otimes n}$ satisfies property $N_{p}$.

Proof. The first statement is a straightforward consequence of the theorem. By Lemma 2.7, the line bundle $A^{\otimes 2}$ is base-point-free, so if $n$ is even the second statement follows from the first. If $n$ is odd the result follows from a slight variation of the argument in the proof of Theorem 2.14: we break up $K_{X} \otimes A^{\otimes n}$ as tensor product of $n-1$ copies of $B=A^{\otimes 2}$ and $B^{\prime}=A^{\otimes 3}$, which is base-point-free by Lemma 2.7. When applying Observation 1.4.1 we take the last map among the $\alpha_{i}$ to be precisely the map involving $B^{\prime}$. The reader can easily verify that the vanishings needed in order to apply (1.4.2) follow by induction or, eventually, by Kodaira Vanishing Theorem.

\section{Koszul rings of Enriques surfaces}

We have devoted Section 2 to the study of syzygies of embeddings of Enriques surfaces. We show in particular a result, Theorem 2.11, about normal presentation of line bundles which were the tensor product of two base-point-free line bundles. Recall that the normal presentation property means that the homogeneous ideal of the (projectively normal) variety is generated by forms of degree 2 . As already pointed out in the introduction, an interesting algebraic property that many normally presented rings have is the Koszul property. There exist many significant examples: canonical rings of curves (cf. [FV], [PP]), rings of curves of degree greater than or equal to $2 g+2$ (cf. [Bu], [GP1]), elliptic ruled surfaces (cf. [GP1], Theorem 5.8) and those line bundles on Enriques surfaces which are normally presented according to Theorem 2.1 (cf. [GP1], Corollary 5.7). This section provides yet one more case in favor of this philosophy: we will show in Theorem 3.5 that those line bundles on an Enriques surface which are normally presented according to Theorem 2.11 also satisfy the Koszul property. Moreover, in the course of proving the result, it can be seen how the property $N_{1}$ is one of the first conditions required for the ring to be Koszul.

To begin we recall some notation and some basic definitions: given a line bundle $L$ on a variety $X$, we set $R(L)=\bigoplus_{n=0}^{\infty} H^{0}\left(X, L^{\otimes n}\right)$. 
Definition 3.1. Let $R=k \oplus R_{1} \oplus R_{2} \oplus \ldots$ be a graded ring and $k$ a field. $R$ is a Koszul ring iff $\operatorname{Tor}_{i}^{R}(\boldsymbol{k}, \boldsymbol{k})$ has pure degree $i$ for all $i$.

We recall now a cohomological interpretation, due to Lazarsfeld, of the Koszul property for a coordinate ring $R(L)$. Let $L$ be a globally generated line bundle on a variety $X$. We will denote $M^{0, L}:=L$ and $M^{1, L}:=M_{L} \otimes L=M_{M^{0, L}} \otimes L$. If $M^{1, L}$ is globally generated, we denote $M^{2, L}:=M_{M^{1, L}} \otimes L$. We repeat the process and define inductively $M^{h, L}:=M_{M^{h-1, L}} \otimes L$, if $M^{h-1, L}$ is globally generated. Now we are ready to state the following slightly modified version of [P1], Lemma 1 :

Lemma 3.2. Let $X$ be a projective variety over an algebraic closed field $\boldsymbol{k}$. Let $L$ be an ample and base-point-free line bundle on $X$. Then $R(L)$ is Koszul iff $M^{h, L}$ exists, is globally generated and $H^{0}\left(M^{h, L}\right) \otimes H^{0}\left(L^{\otimes s+1}\right) \rightarrow H^{0}\left(M^{h, L} \otimes L^{\otimes s+1}\right)$ is surjective for all $h \geqq 0, s \geqq 0$. If, in addition, $H^{1}\left(L^{\otimes s+1}\right)=0$ for every $s \geqq 0$, then $R(L)$ is Koszul iff $H^{1}\left(M^{(h), L} \otimes L^{\otimes s}\right)=0$ and every $s \geqq 0$.

The proof of Theorem 3.5 will follow the same strategy of Section 2, i.e., we will translate the problem in terms of a question about vector bundles over a suitable curve $C$ of $X$. For that purpose we need now a way to relate $M^{(h), L}$ to $M^{(h), L \otimes 0 c}$. We carry this out link by link:

Definition 3.3. Let $X$ be a variety, let $L$ be a line bundle on $X$ and let $b$ be a (smooth) effective divisor on $X$. Assume that $M^{h^{\prime}, L}$ is defined for all $h \geqq h^{\prime} \geqq 0$ (i.e., inductively, $M^{h^{\prime}-1, L}$ is defined and globally generated). We then define, for all $0 \leqq h^{\prime} \leqq h$,

$$
M_{h^{\prime}, \mathrm{b}}^{h^{\prime} L}=M^{h^{\prime}, L} \otimes \mathcal{O}_{\mathrm{b}}
$$

Then $M_{h^{\prime}, \mathrm{b}}^{h^{\prime}, L}$ is globally generated and we define $M_{h^{\prime}, \mathrm{b}}^{h^{\prime}+1, L}=M_{M_{h^{\prime}, \mathrm{b}}^{h^{\prime},}} \otimes L$. If $M_{h^{\prime}, \mathrm{b}}^{h^{\prime}+1, L}$ is again globally generated we define $M_{h^{\prime}, \mathrm{b}}^{h^{\prime}+2, L}=M_{M_{h^{\prime}, b}^{h^{\prime}+1, L}} \otimes L$ and so on.

Lemma 3.4. Let $X$ be a variety, let $\mathfrak{b}$ be a (smooth) effective divisor on $X$ and let $B=\mathcal{O}(\mathfrak{b})$. Let $L$ be a base-point-free line bundle on $X$ such that $M^{h^{\prime} L}$ is globally generated and $H^{1}\left(M^{h^{\prime} L} \otimes B^{*}\right)=0$ for all $0 \leqq h^{\prime} \leqq h-1, H^{1}\left(L \otimes \mathcal{O}_{b}\right)=0$, and $L \otimes \mathcal{O}_{b}$ is Koszul. Then,

(1) $M_{h^{\prime}, \mathrm{b}}^{h, L}$ is globally generated for all $0 \leqq h^{\prime} \leqq h$,

(2) $H^{1}\left(M_{h^{\prime}, \mathbf{b}}^{h, L}\right)=0$ for all $0 \leqq h^{\prime} \leqq h$,

(3) $0 \rightarrow H^{0}\left(M^{h^{\prime}-1, L} \otimes B^{*}\right) \otimes M_{0, \mathrm{~b}}^{h-h^{\prime}, L} \rightarrow M_{h^{\prime}, \mathrm{b}}^{h, L} \rightarrow M_{h^{\prime}-1, \mathrm{~b}}^{h, L} \rightarrow 0$, for all $1 \leqq h^{\prime} \leqq h$.

Proof. The proof is by induction on $h$. If $h=0$, the result is part of the hypotheses. If $h=1$, the exact sequence in (3) is (2.9.1) when we set $F=L$ and twist by $L$. Let us write $L_{\mathfrak{b}}=L \otimes \mathcal{O}_{\mathfrak{b}}$. Since $H^{1}\left(L_{\mathfrak{b}}\right)=0$ and $L_{\mathfrak{b}}$ is Koszul, $H^{1}\left(M_{0, b}^{1, L}\right)=0$, therefore using (3) we obtain indeed that $H^{1}\left(M_{1, \mathfrak{b}}^{1, L}\right)=0$. The bundle $M_{0, \mathfrak{b}}^{1, L}$ is globally generated because $L_{\mathfrak{b}}$ is Koszul. Finally the fact that $M_{1, b}^{1, L}$ is globally generated follows again from (3): we have the following exact commutative diagram 


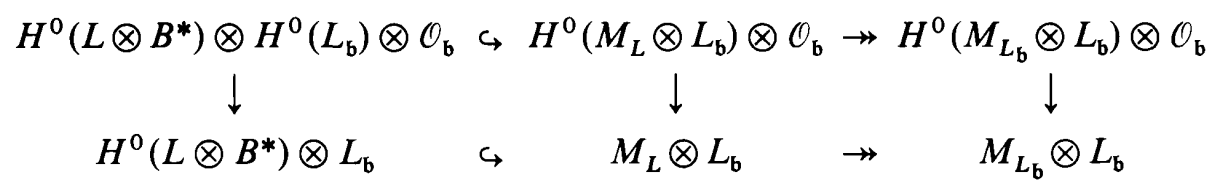

in which the vertical side arrows are surjective because $L_{\mathrm{b}}$ and $M_{L_{\mathrm{b}}} \otimes L_{\mathrm{b}}=M_{0, \mathrm{~b}}^{1, L}$ are both globally generated. Let us now assume the result to be true for $h-1$ and prove it for $h$. We again prove (3) first. If $h=h^{\prime}$, again (3) is nothing but (2.9.1), setting $F=M^{h-1, L}$ (which we know by induction hypothesis to be globally generated) and twisted by $L$. If $h>h^{\prime}$, by induction on $h$ we have the sequence

$$
0 \rightarrow H^{0}\left(M^{h^{\prime}-1, L} \otimes B^{*}\right) \otimes M_{0, \mathrm{~b}}^{h-h^{\prime}-1, L} \rightarrow M_{h^{\prime}, \mathrm{b}}^{h-1, L} \rightarrow M_{h^{\prime}-1, \mathrm{~b}}^{h-1, L} \rightarrow 0 .
$$

Call $V=H^{0}\left(M^{h^{\prime}-1, L} \otimes B^{*}\right)$. Taking global sections, we build this exact commutative diagram:

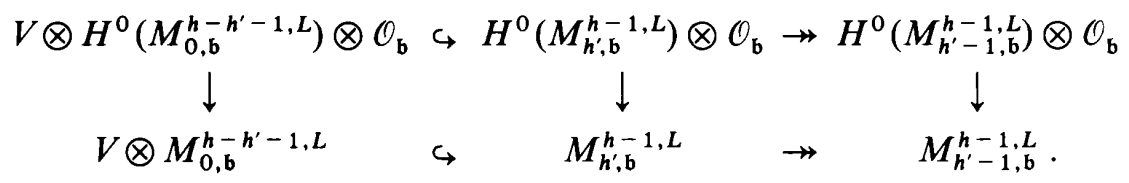

The top horizontal sequence is exact at the right because $H^{1}\left(M_{0, b}^{h-h^{\prime}-1, L}\right)=0$, by induction hypothesis. The vertical arrows are surjective because the vector bundles involved are globally generated by induction hypothesis on $h$. The short exact sequence of kernels is then, after tensoring by $L_{b}$, the sequence wanted for (3). To prove (2), we use induction on $h^{\prime}$. If $h^{\prime}=0$ both (1) and (2) follow from the fact that $L_{\mathfrak{b}}$ is Koszul and $H^{1}\left(L_{\mathfrak{b}}\right)=0$. Now assume that (1) and (2) hold for $h^{\prime}-1$. Condition (2) is a straightforward consequence of already proven (3) and induction hypothesis on both $h$ and $h^{\prime}$. For (1) we use induction on both $h$ and $h^{\prime}$ and (3) just proven. If $h=0$ the surjectivity just follows from the fact that $L_{\mathfrak{b}}$ is Koszul, hence normally generated. If $h^{\prime}=0$ the surjectivity just follows from the fact that $L_{\mathfrak{b}}$ is Koszul. Assume now that the claim holds for $h^{\prime}-1$. The surjectivity of the map for $h^{\prime}$ follows then by chasing the commutative diagram of multiplication maps, built upon (3), having in account the vanishing of $H^{1}\left(M_{0, b}^{h-h^{\prime}, L}\right)$, which follows from (2), and the surjectivity of the vertical side maps, which follows from induction hypothesis on $h$ and $h^{\prime}$. Then the fact that $L_{\mathrm{b}}$ is ample implies the global generation of $M_{h^{\prime}, \mathrm{b}}^{h, L}$ as wished.

We are now ready to prove the main theorem of this section:

Theorem 3.5. Let $X$ be an Enriques surface. Let $B_{1}$ and $B_{2}$ be ample and base-point-free line bundles, such that $B_{1} \cdot B_{2} \geqq 6$. If $L=B_{1} \otimes B_{2}$, then $R(L)$ is Koszul. and that

Proof. According to Lemma 3.2 we need to show that $M^{h, L}$ is globally generated

$$
H^{0}\left(M^{h, L}\right) \otimes H^{0}\left(L^{\otimes s}\right) \stackrel{\alpha}{\longrightarrow} H^{0}\left(M^{h, L} \otimes L^{\otimes s}\right)
$$

surjects for all $h \geqq 0$ and $s \geqq 1$. To better carry out the argument, is convenient to also prove $H^{1}\left(M^{h, L} \otimes B_{1}^{*}\right)=H^{1}\left(M^{h, L} \otimes B_{2}^{*}\right)=0$. The proof is by induction on $h$. If $h=0$ the 
result is the projective normality of $L=B_{1} \otimes B_{2}$, which follows from Theorem 2.2, and Kodaira vanishing. Now assume the result for $h-1$. Since $L$ is ample, the surjectivity of $\alpha$ implies the global generation of $M^{h, L}$, hence we can assume that $M^{h^{\prime}, L}$ is globally generated for all $0 \leqq h^{\prime}<h$ and we need only to prove that $\alpha$ surjects and that

$$
H^{1}\left(M^{h, L} \otimes B_{1}^{*}\right)=H^{1}\left(M^{h, L} \otimes B_{2}^{*}\right)=0 .
$$

We start proving the former and in the course of the proof we will also obtain the desired vanishings. According to Observation 1.4.1 we are done if we prove that certain collection of multiplication maps surject. We prove the surjectivity of the first of them, which is

$$
H^{0}\left(M^{h, L}\right) \otimes H^{0}\left(B_{1}\right) \stackrel{\beta}{\longrightarrow} H^{0}\left(M^{h, L} \otimes B_{1}\right) .
$$

The argument to prove the surjectivity of the rest is analogous. We prove it using again induction on $h$. We proved the statement for $h=0$ in the course of proving the projective normality of $L$ in Theorem 2.2. Assume the statement to be true for $h-1$ (we may also assume the surjectivity of the map $\beta$ for $h-1$ if we substitute in the formula $B_{1}$ by $B_{2}$, since the roles of $B_{1}$ and $B_{2}$ are interchangeable. Consider the sequence

$$
\begin{gathered}
H^{0}\left(M^{h-1, L}\right) \otimes H^{0}\left(B_{2}\right) \stackrel{\gamma}{\longrightarrow} H^{0}\left(M^{h-1, L} \otimes B_{2}\right) \\
\rightarrow H^{1}\left(M^{h, L} \otimes B_{1}^{*}\right) \rightarrow H^{1}\left(M^{h-1, L}\right) \otimes H^{0}\left(B_{2}\right) .
\end{gathered}
$$

The multiplication map $\gamma$ is surjective by induction hypothesis. The group $H^{1}\left(M^{h-1, L}\right)$ vanishes also by induction hypothesis, therefore $H^{1}\left(M^{h, L} \otimes B_{1}^{*}\right)=0$. On the other hand $H^{1}\left(\mathcal{O}_{X}\right)=0$, so in order to see the surjectivity of $\beta$ it is enough to check the surjectivity of

$$
H^{0}\left(M_{h, b_{1}}^{h, L}\right) \otimes H^{0}\left(B_{1} \otimes \mathcal{O}_{b_{1}}\right) \stackrel{\delta}{\longrightarrow} H^{0}\left(M_{h, b_{1}}^{h, L} \otimes B_{1}\right),
$$

where $b_{1}$ is a smooth irreducible curve in $\left|B_{1}\right|$. To see the surjectivity of $\delta$ we will use Lemma 3.4 inductively on $h^{\prime}$. More precisely we want to prove that

$$
H^{0}\left(M_{h, b_{1}}^{h, L}\right) \otimes H^{0}\left(B_{1} \otimes \mathcal{O}_{b_{1}}\right) \rightarrow H^{0}\left(M_{h, b_{1}}^{h, L} \otimes B_{1}\right)
$$

surjects for all $0 \leqq h^{\prime} \leqq h$. If $h^{\prime}=0, M_{0, b_{1}}^{h, L}$ is semistable with slope strictly bigger than $2 g+2$ by Lemma 2.10, hence by Proposition 2.4 the multiplication map in question is surjective. Now assume the statement to be true for $h^{\prime}-1$. We take global sections in the sequence in part (3) of the statement of Lemma 3.4 and tensor with $U=H^{0}\left(B_{1} \otimes \mathcal{O}_{\mathbf{b}_{1}}\right)$ to obtain the following exact commutative diagram:

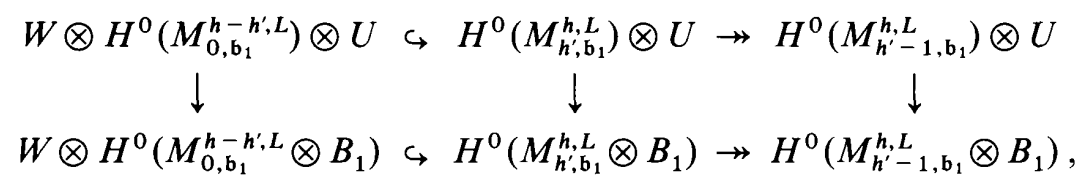

where $W=H^{0}\left(M^{h^{\prime}-1, L} \otimes B_{1}\right)$. The surjectivity of the left hand side vertical map and the exactness at the right of the top horizontal sequence follow both from Proposition 2.4 and Lemma 2.10. The surjectivity of the right hand side vertical map follows by the induction hypothesis on $h^{\prime}$. 


\section{Abelian and bielliptic surfaces}

In this section we deal with the remaining classes of surfaces with Kodaira dimension 0 , namely, those with nonzero irregularity. For the techniques employed we return to those used in the arguments of Section 1. The main theorem we will prove is

Theorem 4.1. Let $X$ be an Abelian or a bielliptic surface. Let $B$ be an ample and base-point-free line bundle with $B^{2} \geqq 5$ and let $N$ be a numerically trivial line bundle on $X$. Let $L_{1} \equiv B^{\otimes l_{1}+1}$ and $L_{2} \equiv B^{\otimes l_{2}+1}$. If $l_{1}, l_{2} \geqq p \geqq 1$, then

$$
H^{1}\left(M_{L_{1}}^{\otimes p+1} \otimes L_{2}\right)=H^{1}\left(M_{L_{1}} \otimes L_{2}\right)=0
$$

In particular, if $n \geqq p \geqq 1$, then $B^{n+1} \otimes N$ satisfies the property $N_{p}$.

Before we prove Theorem 4.1 we need the following

Lemma 4.2. Let $X$ be a surface with $\kappa=0$. Let $B$ be an ample and base-point-free line bundle. Let $L_{1}=B_{1}^{1} \otimes \cdots \otimes B_{l_{1}}^{1}$, where $B_{i}^{1} \equiv B$ are base-point-free line bundles and $l_{1} \geqq 1$ and $L_{2}=B_{1}^{2} \otimes \cdots \otimes B_{l_{2}}^{2}$, where $B_{j}^{2} \equiv B$ and $l_{2} \geqq 1$. If either

(1) $l_{1}$ or $l_{2}$ are greater than or equal to 3 or,

(2) $l_{1}=2, l_{2}=1$ or 2 and $H^{2}\left(L_{1} \otimes\left(B_{1}^{2}\right)^{-2}\right)=0$ or,

(3) $X$ is Abelian or bielliptic surface, $B^{2} \geqq 5$, and $l_{1}=l_{2}=2$,

then $H^{1}\left(M_{L_{1}} \otimes L_{2}\right)=0$.

Proof. In cases (1) and (2) the result follows from iteratively applying (1.4.2) using Observation 1.4.1 and Kodaira vanishing. In case (3), let us write $L_{1}$ as $B^{\otimes 2} \otimes E_{1}$ with $E_{1} \equiv 0$. We can find $E \equiv 0$ with $E^{\otimes 2} \neq E_{1} \otimes K^{*}$, because not all elements in $\operatorname{Pic}^{0}(X)$ have order 2. Then, by Lemma 2.13, we can assume that $B_{1}^{2}=B \otimes E$. Then

$$
H^{2}\left(L_{1} \otimes\left(B_{1}^{2}\right)^{-2}\right)=H^{0}\left(K \otimes E^{\otimes 2} \otimes E_{1}^{*}\right)=0,
$$

which follows from our choice of $E$, and we are in case (2).

(4.3) Proof of Theorem 4.1. The vanishing of $H^{1}\left(M_{L_{1}} \otimes L_{2}\right)$ is a consequence of Lemma 4.2. The proof of the vanishings of $H^{1}\left(M_{L_{1}}^{\otimes p+1} \otimes L_{2}\right)$ is by induction. As usual the key step is the first: $p=1$. We need to prove that $H^{1}\left(M_{R_{1}}^{\otimes 2} \otimes R_{2}\right)=0$ if $R_{1} \equiv B^{\otimes r_{1}}$ and $R_{2} \equiv B^{\otimes r_{2}}$ and $r_{1}, r_{2} \geqq 2$. Using the sequence (1.1) we obtain

$$
\begin{gathered}
H^{0}\left(M_{R_{1}} \otimes R_{2}\right) \otimes H^{0}\left(R_{1}\right) \stackrel{\alpha}{\longrightarrow} H^{0}\left(M_{R_{1}} \otimes R_{1} \otimes R_{2}\right) \\
\rightarrow H^{1}\left(M_{R_{1}}^{\otimes 2} \otimes R_{2}\right) \rightarrow H^{1}\left(M_{R_{1}} \otimes R_{2}\right) \otimes H^{0}\left(R_{1}\right)
\end{gathered}
$$


The group $H^{1}\left(M_{R_{1}} \otimes R_{2}\right)$ vanishes by Lemma 4.2. Therefore the sought vanishing is equivalent to the surjectivity of $\alpha$. If $r_{1} \geqq 3$, let $B_{1}^{1}=B$. If $r_{1}=2$, let $R_{1}=B^{\otimes 2} \otimes E_{1}$. Analogously, if $r_{2}=2$, let $R_{2}=B^{\otimes 2} \otimes E_{2}$. We may now assume if $r_{1} \geqq 2$, by Lemma 2.13, that $B_{1}^{1}=B \otimes E$ with $E \equiv 0$ but $E^{\otimes 2} \neq K^{*} \otimes E_{2}$ and, if in addition $r_{2}=2$, that

$$
E^{\otimes 2} \neq K \otimes E_{2}^{\otimes 2} \otimes E_{1}^{*}
$$

also. We can always find such an $E$ if not all elements in $\operatorname{Pic}^{0}(X)$ have order 2,4 or 6 . This is the case for Abelian and bielliptic surfaces, which possess numerically trivial line bundles of infinite order. Then, to see that $\alpha$ surjects, by (1.4.2) and Observation 1.4.1 it suffices to check that

$$
\begin{gathered}
H^{1}\left(M_{R_{1}} \otimes R_{2} \otimes\left(B_{1}^{1}\right)^{*}\right)=0, \\
H^{2}\left(M_{R_{1}} \otimes R_{2} \otimes\left(B_{1}^{1}\right)^{-2}\right)=0, \\
H^{1}\left(M_{R_{1}} \otimes R_{2}^{\prime} \otimes B^{\otimes \gamma}\right)=0 \quad \text { for all } 0 \leqq \gamma \text { and } R_{2}^{\prime} \equiv R_{2}, \\
H^{2}\left(M_{R_{1}} \otimes R_{2}^{\prime \prime} \otimes B^{\otimes \gamma-1}\right)=0 \quad \text { for all } 0 \leqq \gamma \text { and } R_{2}^{\prime \prime} \equiv R_{2} .
\end{gathered}
$$

The vanishing (4.3.4) follows from (1.1) and Kodaira Vanishing Theorem. The vanishing (4.3.2) follows from (1.1), Kodaira Vanishing Theorem and the way in which we have chosen $E$. The vanishing in (4.3.3) follows from Lemma 4.2. Finally, (4.3.1) follows from Lemma 4.2 once we see that if $r_{1}=r_{2}=2$,

$$
H^{2}\left(R_{1} \otimes R_{2}^{-2} \otimes\left(B_{1}^{1}\right)^{\otimes 2}\right)=H^{2}\left(E_{1} \otimes E^{\otimes 2} \otimes E_{2}^{-2}\right)=0,
$$

which follows from the way in which we have chosen $E$.

Assume the result true for $p-1$ and $p>1$. We have the following sequence:

$$
\begin{gathered}
H^{0}\left(M_{R_{1}}^{\otimes p} \otimes R_{2}\right) \otimes H^{0}\left(R_{1}\right) \stackrel{\beta}{\longrightarrow} H^{0}\left(M_{R_{1}}^{\otimes p} \otimes R_{1} \otimes R_{2}\right) \\
\rightarrow H^{1}\left(M_{R_{1}}^{\otimes p+1} \otimes R_{2}\right) \rightarrow H^{1}\left(M_{R_{1}}^{\otimes p} \otimes R_{2}\right) \otimes H^{0}\left(R_{1}\right) .
\end{gathered}
$$

The last term is zero by induction hypothesis, so the desired vanishing is equivalent to the surjectivity of $\beta$. This follows from Observation 1.4.1 and (1.4.2). In fact, the required vanishings follow by induction, Kodaira Vanishing Theorem and Lemma 4.2.

For the last conclusion of the theorem, note that

$$
H^{1}\left(M_{L}^{\otimes p^{\prime}+1} \otimes L^{\otimes s}\right)=0 \quad \text { for all } p \geqq p^{\prime} \geqq 0 \text { and all } s \geqq 1 .
$$

Then, by Theorem 1.2, $L$ satisfies property $N_{p}$.

Either as straightforward consequence of Theorem 4.1 or from the same ideas we have been using we obtain results for adjoint linear series: 
Corollary 4.4. Let $X$ be an Abelian or bielliptic surface. Let $B$ be an ample and base-point-free line bundle such that $B^{2} \geqq 5$. Then $K \otimes B^{\otimes n}$ satisfies property $N_{p}$ if $n \geqq p+1$, $p \geqq 1$.

Corollary 4.4 implies Mukai's conjecture for Abelian and bielliptic surfaces and $p=0$, and for $p=1$ (in the latter case, our result improves Mukai's bound):

Corollary 4.5. Let $X$ be an Abelian or a bielliptic surface. Let $A$ be an ample line bundle and $L=K_{X} \otimes A^{\otimes n}$. If $n \geqq 2 p+2$ and $p \geqq 1$, then $L$ satisfies property $N_{p}$. In particular, if $n \geqq 4$, L satisfies property $N_{1}$.

Proof. $A^{\otimes 2}$ is base-point-free by Lemma 2.7 (for Abelian surfaces this also follows from Lefschetz's Theorem) and since $K \equiv 0, A^{2} \geqq 2$ and $\left(A^{\otimes 2}\right)^{2} \geqq 8$. Then, if $n$ is even the result is a straightforward consequence of Corollary 4.4. If $n$ is odd the situation is the same as that of Corollary 2.15 and we proceed analogously.

Remark 4.6. If $X$ is an Abelian surface the above result was proven by Kempf (cf. $[\mathrm{Ke}])$. However, the results proven in this chapter are more general: for instance, since on an Abelian surface a polarization of type $(1,3)$ is base-point-free, Corollary 4.4 implies that a line bundle of type $(p+1,3 p+3)$ satisfies property $N_{p}$. This fact does not follow from Kempf's result.

To end this section we carry out a study analogous to the one realized for Enriques surfaces in Section 3: the following theorem proves in particular that the line bundles satisfying property $N_{1}$ according to Theorem 4.1 have also a Koszul coordinate ring.

Theorem 4.7. Let $X$ be an Abelian or a bielliptic surface. Let $B_{1}$ and $B_{2}$ be numerically equivalent ample and base-point-free line bundles with self-intersection bigger than or equal to 5. If $L=B_{1} \otimes B_{2}$, then $R(L)$ is Koszul. In particular $L$ satisfies property $N_{1}$.

In order to prove the theorem we use the following result which is basically a reformulation of [GP1], Theorem 5.4 for the case of surfaces with $\kappa=0$ :

Lemma 4.8. Let $X$ be a surface with $\kappa=0$, let $B_{1}$ and $B_{2}$ be two ample and base-pointfree line bundles. If $H^{2}\left(B_{1} \otimes B_{2}^{*}\right)=H^{2}\left(B_{2} \otimes B_{1}^{*}\right)=0$, then the following properties are satisfied for all $h \geqq 0$ :

(1) $M^{h, L}$ is globally generated,

(2) $H^{1}\left(M^{h, L} \otimes B_{1}^{\otimes b_{1}} \otimes B_{2}^{\otimes b_{2}}\right)=0$ for all $b_{1}, b_{2} \geqq 0$,

(3) $H^{1}\left(M^{h, L} \otimes B_{j}^{*}\right)=0$ where $j=1,2$,

(4) $H^{1}\left(M^{h, L} \otimes B_{i} \otimes B_{j}^{*}\right)=0$ where $i=1,2$ and $j=2,1$,

(5) $H^{1}\left(M^{h, L} \otimes B_{i}^{\otimes 2} \otimes B_{j}^{*}\right)=0$ where $i=1,2$ and $j=2,1$.

In particular $H^{1}\left(M^{(h), L} \otimes L^{\otimes s}\right)=0$ for all $h, s \geqq 0$, and $R(L)$ is a Koszul $k$-algebra. 
Proof. For the proof of the lemma we refer to [GP1]. Since now $B_{1}$ and $B_{2}$ are ample and $K_{X} \equiv 0$, we obtain all the vanishings of the groups $H^{1}\left(B_{1}^{\otimes a} \otimes B_{2}^{\otimes b}\right)$ when $a, b \geqq 0$ and $a+b \geqq 1$ needed in the proof, by Kodaira Vanishing Theorem, making therefore unnecessary to assume the vanishings of $H^{1}\left(B_{1}\right), H^{1}\left(B_{2}\right)$ and $H^{2}\left(\mathcal{O}_{X}\right)$.

(4.9) Proof of Theorem 4.7. The result follows from Lemma 4.8. If $X$ is bielliptic, the only problem we might have is if $B_{1}=B_{2} \otimes K_{X}$ or if $B_{2}=B_{1} \otimes K_{X}$. In the former case, choose a line bundle $E \in \mathrm{Pic}^{0}(X)$ such that $E^{-2} \neq 0$ and $K_{X}^{\otimes 2} \otimes E^{\otimes 2} \neq 0$. In the latter case choose $E$ such that $E^{\otimes 2} \neq 0$ and $K_{X}^{\otimes 2} \otimes E^{-2} \neq 0$. Let then $B_{1}^{\prime}=B_{1} \otimes E$ and $B_{2}^{\prime}=B_{2} \otimes E^{*}$. The desired result follows if we apply Lemma 4.8 to $B_{1}^{\prime}$ and $B_{2}^{\prime}$ instead.

If $X$ is an Abelian surface, $K_{X}$ is trivial, so the only problem applying Lemma 4.8 would appear when $B_{1}=B_{2}$. This is solved analogously considering $B_{1}^{\prime}=B_{1} \otimes E$ and $B_{2}^{\prime}=B_{2} \otimes E^{*}$, where now $E$ is taken to have nontrivial square.

\section{Surfaces of positive Kodaira dimension}

In this section we focus on the study of adjoint linear series of surfaces of positive Kodaira dimension. We find sufficient conditions for the normal generation and the normal presentation of the adjoint linear series and of the powers of an ample and base-point-free line bundle. For the latter case we also generalize the results to higher syzygies. One can look upon these results as an analogue for projective normality and higher syzygies of the results of Kawamata and Shokurov (see [Ka] and [Sh]) for base-point-freeness and effectiveness, viewed in the special context of algebraic surfaces. They deal with nef bundles $L$ for which $L \otimes K^{*}$ is nef and big and conclude the freeness and effectiveness of multiples of $L$. We start with an ample and base-point-free bundle $B$ which satisfies certain inequalities (see Theorems 5.1 and 5.8) which are immediate if one assumes that $B \otimes K_{S}^{*}$ is nef and big and go on to prove projective normality and higher syzygy results for powers of $B$ and for adjunction bundles associated to $B$.

We obtain two interesting consequences from our study. The first is finding sufficient conditions for projective normality and quadratic generation of pluricanonical embeddings of surfaces of general type (Corollary 5.6, Remark 5.7 and Corollary 5.9). Bombieri asked in [Bo] whether $\left|K_{S}^{\otimes 5}\right|$ maps $S$ as a projectively normal variety. This question was answered affirmatively by Ciliberto in [Ci] (under basically the same assumptions of Theorem 5.5 below). Thus the above mentioned corollaries recover, and in the case of regular surfaces, improve Ciliberto's result. The second consequence is an effective bound along the lines of Mukai's conjecture using a result by Fernández del Busto. In the case of pluricanonical models of regular surfaces of general type we further our study to higher syzygies. Ein and Lazarsfeld's results in [EL] together with Del Busto's give effective bounds (slightly weaker than ours) along the lines of the Mukai's conjecture, but for regular surfaces, the bounds we obtain are better. We also obtain as a corollary of Theorem 5.1 (4) effective bounds for property $N_{p}$ for the multiples of ample line bundles on regular surfaces.

Theorem 5.1. Let $S$ be a regular surface of positive Kodaira dimension and $p_{g} \geqq 4$. Let $B$ be an ample and base-point-free line bundle such that $H^{1}(B)=0$. Let $L=K_{S} \otimes B^{\otimes n}$ and $L^{\prime}=K_{S} \otimes B^{\otimes l}$. Let $N=B^{\otimes m}$ and $N^{\prime}=B^{\otimes k}$. 
(1) If $\kappa(S)=1$ and $B^{2}>K_{S} \cdot B$, and if $n, l \geqq 2$, then

$$
H^{1}\left(M_{L} \otimes L^{\prime}\right)=H^{1}\left(M_{L}^{\otimes 2} \otimes L^{\prime}\right)=0 .
$$

In particular, $K_{S} \otimes B^{\otimes n}$ satisfies property $N_{1}$, for all $n \geqq 2$.

(2) If $\kappa(S)=2$ and $B^{2} \geqq K_{S} \cdot B$, and if $n, l \geqq 2$, then $H^{1}\left(M_{L} \otimes L^{\prime}\right)=0$. In particular, $K_{S} \otimes B^{\otimes n}$ satisfies property $N_{0}$, for all $n \geqq 2$.

(3) If $\kappa(S)=2$ and $B^{2} \geqq 2 K_{S} \cdot B$, and if $n, l \geqq 2$, then

$$
H^{1}\left(M_{L} \otimes L^{\prime}\right)=H^{1}\left(M_{L}^{\otimes 2} \otimes L^{\prime}\right)=0 ;
$$

and if $m, k \geqq 2$, then $H^{1}\left(M_{N} \otimes N^{\prime}\right)=H^{1}\left(M_{N}^{\otimes 2} \otimes N^{\prime}\right)=0$. In particular $K_{S} \otimes B^{\otimes n}$ and $B^{\otimes m}$ satisfy property $N_{1}$, for all $n, m \geqq 2$.

(4) If $\kappa(S)=2$ and $B^{2} \geqq 2 K_{S} \cdot B$, and if $m, k \geqq p+1, p \geqq 1$, then

$$
H^{1}\left(M_{N}^{\otimes p+1} \otimes N^{\prime}\right)=0 .
$$

In particular if $p \geqq 1, B^{\otimes m}$ satisfy property $N_{p}$, for all $m \geqq p+1$.

To prove Theorem 5.1 we will need these two lemmas:

Lemma 5.2. Let $S$ be a surface and $B$ an ample and base-point-free line bundle with $H^{1}(B)=0$ and $B^{2}>B \cdot K_{S}$ if $\kappa(S) \leqq 1$, and $B^{2} \geqq B \cdot K_{S}$ if $\kappa(S)=2$. Then $H^{1}\left(B^{\otimes m}\right)=0$ for all $m \geqq 1$.

Proof. Let $C$ be a smooth curve in $|B|$. Since $\operatorname{deg}\left(B^{\otimes m} \otimes \mathcal{O}_{C}\right)>2 g(C)-2$ when $m \geqq 3$, we only have to prove $H^{1}\left(B^{\otimes 2}\right)=0$. If $B^{2}>B \cdot K_{S}$ or $B^{\otimes 2} \otimes \mathcal{O}_{C} \neq K_{C}$, then $H^{1}\left(B^{\otimes 2} \otimes \mathcal{O}_{C}\right)=0$, hence $H^{1}\left(B^{\otimes 2}\right)=0$ because $H^{1}(B)=0$. If $B^{\otimes 2} \otimes \mathcal{O}_{C}=K_{C}$, then $B \otimes \mathcal{O}_{C}=K_{S} \otimes \mathcal{O}_{C}$. Consider the sequence

$$
0 \rightarrow H^{0}\left(K_{S}^{*}\right) \rightarrow H^{0}\left(B \otimes K_{S}^{*}\right) \rightarrow H^{0}\left(B \otimes K_{S}^{*} \otimes \mathcal{O}_{C}\right) \rightarrow H^{1}\left(K_{S}^{*}\right) .
$$

Since in this case $S$ is a surface of general type, $H^{0}\left(K_{S}^{*}\right)=H^{1}\left(K_{S}^{*}\right)=0$, therefore $B \otimes K_{S}^{*}$ is effective and since $B$ is ample, it must be $B \otimes K_{S}^{*}=\mathcal{O}_{S}$. Hence

$$
H^{1}\left(B^{\otimes 2}\right)=H^{1}\left(K_{S}^{\otimes 2}\right)=0 .
$$

Lemma 5.3. Let $S$ be an algebraic surface with nonnegative Kodaira dimension and let $B$ be an ample line bundle. Let $m \geqq 1$. If $B^{2} \geqq m K_{S} \cdot B$, then $K_{S} \cdot B \geqq m K_{S}^{2}$.

Proof. We assume the contrary, i.e., that $K_{S} \cdot B<m K_{S}^{2}$, and get a contradiction. Let $L=B \otimes K_{S}^{-m}$. We have that $L^{2}>0$. By Riemann-Roch

$$
h^{0}\left(L^{\otimes n}\right) \geqq \frac{n^{2} L^{2}-n K_{S} \cdot L}{2}+\chi\left(\mathcal{O}_{S}\right)-h^{0}\left(K_{S} \otimes L^{-n}\right) .
$$


If $B^{2}>m K_{S} \cdot B,\left(K_{S} \otimes L^{\otimes-n}\right) \cdot B<0$, for $n$ large enough, and since $B$ is ample, $K_{S} \otimes L^{\otimes-n}$ is not effective, so finally $L^{\otimes n}$ is effective for $n$ large enough. But in that case $n K_{S} \cdot L \geqq 0$, because $K_{S}$ is nef, contradicting our assumption.

Now if $B^{2}=m K_{S} \cdot B$, we have that $L^{2}>0, B^{2}>0$ (because $B$ is ample), and $L \cdot B=0$, but this is impossible by the Hodge index theorem.

(5.4) Proof of Theorem 5.1. We start proving that $H^{1}\left(M_{L} \otimes L^{\prime}\right)=0$ if $B$ satisfies the conditions of (1) and (2). By Observation 1.4.1 it suffices to show that

$$
\begin{gathered}
H^{0}\left(K_{S} \otimes B^{\otimes n}\right) \otimes H^{0}(B) \rightarrow H^{0}\left(K_{S} \otimes B^{\otimes n+1}\right), \\
H^{0}\left(K_{S} \otimes B^{\otimes m}\right) \otimes H^{0}\left(K_{S} \otimes B\right) \rightarrow H^{0}\left(K_{S} \otimes B^{\otimes m+1}\right), \quad \text { for all } n \geqq 2, m \geqq 3
\end{gathered}
$$

surject. We want to show now that $K_{S} \otimes B$ is base-point-free. Let $C \in|B|$ smooth. From the arguments of the proof of Lemma 5.2, we see that $h^{2}(B) \leqq 1$ with equality if and only if $B=K_{S}$ and $S$ of general type. Since $S$ is regular we have

$$
0 \rightarrow H^{0}\left(\mathcal{O}_{S}\right) \rightarrow H^{0}(B) \rightarrow H^{0}\left(B \otimes \mathcal{O}_{C}\right) \rightarrow 0
$$

Thus it follows from this and from Riemann-Roch that $h^{0}\left(B \otimes \mathcal{O}_{C}\right) \geqq p_{g}-1$ (again, with equality if and only if $B=K_{S}$ and $S$ of general type). Now by Clifford's bound, $B^{2} \geqq 6$ except if $B=K_{S}$ and $S$ of general type. It is well known (cf. Theorem 5.5) that $K_{S}^{\otimes 2}$ is base-point-free under the hypothesis of the theorem. Now, if $B^{2} \geqq 6, K_{S} \otimes B$ is base-pointfree by Lemma 2.13. Take now $C^{\prime} \in\left|K_{S} \otimes B\right|$, also a smooth curve. Since $H^{1}\left(\mathcal{O}_{S}\right)=0$ and by Lemma 5.2 and Kodaira Vanishing Theorem, we may apply Observation 2.3 and conclude that it is enough to check that

$$
\begin{gathered}
H^{0}\left(K_{S} \otimes B^{\otimes n} \otimes \mathcal{O}_{C}\right) \otimes H^{0}\left(B \otimes \mathcal{O}_{C}\right) \rightarrow H^{0}\left(K_{S} \otimes B^{\otimes n+1} \otimes \mathcal{O}_{C}\right), \\
H^{0}\left(K_{S} \otimes B^{\otimes m} \otimes \mathcal{O}_{C^{\prime}}\right) \otimes H^{0}\left(K_{S} \otimes B \otimes \mathcal{O}_{C^{\prime}}\right) \rightarrow H^{0}\left(K_{S} \otimes B^{\otimes m+1} \otimes \mathcal{O}_{C^{\prime}}\right)
\end{gathered}
$$

surject for all $n \geqq 2, m \geqq 3$. This follows from Proposition 2.4 or Proposition 2.5. We check this explicitly for the first family of maps. Let $G=K_{S} \otimes B^{\otimes n} \otimes \mathcal{O}_{C}$ and $G^{\prime}=B \otimes \mathcal{O}_{C}$. It is enough to show that $\operatorname{deg} G>2 g(C)$ and that $\operatorname{deg} G+\operatorname{deg} G^{\prime}>4 g-2 h^{1}\left(G^{\prime}\right)$. For the first inequality, note that $K_{S} \otimes B \otimes \mathcal{O}_{C}=K_{C}$ and that $\operatorname{deg}\left(B \otimes \mathcal{O}_{C}\right) \geqq 4$. For the second,

$$
\text { (5.4.1) } \operatorname{deg} G+\operatorname{deg} G^{\prime} \geqq K_{S} \cdot B+3 B^{2} \geqq 2\left(K_{S} \cdot B+B^{2}\right)=4 g(C)-4 \text {. }
$$

On the other hand $h^{1}\left(G^{\prime}\right)=p_{g}-h^{0}\left(K_{S} \otimes B^{*}\right)$ and since $B$ is ample, the inequality

$$
B^{2} \geqq K_{S} \cdot B
$$

implies $h^{1}\left(G^{\prime}\right) \geqq p_{g}-1$. By the bound on $p_{g}$ we therefore have $4 g-4 \geqq 4 g+2-2 h^{1}\left(G^{\prime}\right)$. The reasoning for the second family of maps is similar. 
We go on now to prove (3). The proof of the vanishing of $H^{1}\left(M_{N} \otimes N^{\prime}\right)$ uses the same above arguments and we will not repeat them here. We now prove the vanishings of $H^{1}\left(M_{L}^{\otimes 2} \otimes L^{\prime}\right)$ and $H^{1}\left(M_{N}^{\otimes 2} \otimes N^{\prime}\right)$. By Observation 1.4.1 it is enough to show that the following maps

$$
\begin{gathered}
H^{0}\left(M_{L} \otimes L^{\prime}\right) \otimes H^{0}(B) \stackrel{\alpha_{1}}{\longrightarrow} H^{0}\left(M_{L} \otimes L^{\prime} \otimes B\right), \\
H^{0}\left(M_{L} \otimes L^{\prime} \otimes B\right) \otimes H^{0}\left(K_{S} \otimes B\right) \stackrel{\alpha_{2}}{\longrightarrow} H^{0}\left(M_{L} \otimes L^{\prime} \otimes K_{S} \otimes B^{\otimes 2}\right), \\
H^{0}\left(M_{N} \otimes N^{\prime}\right) \otimes H^{0}(B) \stackrel{\alpha_{3}}{\longrightarrow} H^{0}\left(M_{N} \otimes N^{\prime} \otimes B\right)
\end{gathered}
$$

surject. We only sketch in some detail the proof of the surjectivity of $\alpha_{1}$, as the proof for the other two maps are analogous. We will use Observation 2.3 and Lemma 2.9. For that we need to check that $H^{1}\left(M_{L} \otimes L^{\prime} \otimes B^{*}\right)=0$. This follows from the surjectivity of

$$
\begin{gathered}
H^{0}(L) \otimes H^{0}(B) \stackrel{\beta_{1}}{\longrightarrow} H^{0}(L \otimes B), \\
H^{0}(L) \otimes H^{0}\left(K_{S} \otimes B\right) \stackrel{\beta_{2}}{\longrightarrow} H^{0}\left(L \otimes K_{S} \otimes B\right) .
\end{gathered}
$$

The surjectivity of $\beta_{1}$ has already been shown previously in this proof. We show now the surjectivity of $\beta_{2}$. Let $C^{\prime} \in\left|K_{S} \otimes B\right|$. By the vanishing of $H^{1}(B)$ from the hypothesis and Kodaira vanishing the surjectivity of $\beta_{2}$ follows from the surjectivity of

$$
H^{0}\left(L \otimes \mathcal{O}_{C^{\prime}}\right) \otimes H^{0}\left(K_{S} \otimes B \otimes \mathcal{O}_{C^{\prime}}\right) \stackrel{\gamma}{\longrightarrow} H^{0}\left(L \otimes K_{S} \otimes B \otimes \mathcal{O}_{C^{\prime}}\right)
$$

by Observation 2.3. We want to apply Proposition 2.5. Note that

$$
\operatorname{deg}\left(L \otimes \mathcal{O}_{C^{\prime}}\right)+\operatorname{deg}\left(K_{S} \otimes B \otimes \mathcal{O}_{C^{\prime}}\right) \geqq 2 K_{S}^{2}+5 K_{S} \cdot B+3 B^{2}
$$

and $4 g\left(C^{\prime}\right)-4=4 K_{S}^{2}+6 K_{S} \cdot B+2 B^{2}$. Since $B^{2} \geqq 2 K_{S} \cdot B$, by Lemma 5.3,

$$
B^{2} \geqq 2 K^{2}+K_{S} \cdot B
$$

Finally $h^{1}\left(K_{S} \otimes B \otimes \mathcal{O}_{C^{\prime}}\right)=p_{g} \geqq 4$, so the inequalities needed to apply Proposition 2.5 are satisfied and the surjectivity of $\gamma$ follows. Returning to the proof of the surjectivity of $\alpha_{1}$, we may now apply Observation 2.3 and Lemma 2.9 and therefore it suffices to check the surjectivity of

$$
H^{0}\left(M_{L \otimes \mathcal{O}_{C}} \otimes L^{\prime} \otimes \mathcal{O}_{C}\right) \otimes H^{0}\left(B \otimes \mathcal{O}_{C}\right) \rightarrow H^{0}\left(M_{L \otimes \mathcal{O}_{C}} \otimes L^{\prime} \otimes B \otimes \mathcal{O}_{C}\right),
$$

which follows from Proposition 2.4. Indeed, let $E=B \otimes \mathcal{O}_{C}$ and $F=M_{L \otimes \mathscr{O}_{C}} \otimes L^{\prime} \otimes \mathcal{O}_{C}$. We need to check that $\mu(F)>2 g(C)$ and that $\mu(F)>2 g+\operatorname{rank}(E)(2 g(C)-\mu(E))-2 h^{1}(E)$. For the former inequality, $\mu(F) \geqq \mu\left(M_{L \otimes \mathcal{O}_{C}}\right)+K_{S} \cdot B+2 B^{2}$ and this is bigger than $2 g(C)$ since $2 g(C)=K_{S} \cdot B+B^{2}+2$ and $B^{2} \geqq 4$. The latter inequality follows from

$$
\operatorname{deg} L \otimes \mathcal{O}_{C}>2 g(C)
$$

as $B^{2} \geqq K_{S} \cdot B, h^{1}\left(B \otimes \mathcal{O}_{C}\right) \geqq 3$ and $B^{2} \geqq 4$. 
Finally the proof of the vanishing of $H^{1}\left(M_{L}^{\otimes 2} \otimes L^{\prime}\right)$ if $\kappa(S)=1$ follows by the same arguments and it is left to the reader.

The proof (4) is built upon (3), using induction on $p$ and (1.4.2).

We want now to apply this theorem to the study of pluricanonical models of surfaces of general type (regular, for the moment; we will complete the picture when we have at our disposal Theorem 5.8 which deals with irregular surfaces). The idea is to find a smallest power of $K_{S}$ which is base-point-free, for it would play the role of $B$. It is known that under certain mild conditions $K_{S}^{\otimes n}$ is base-point-free if $n \geqq 2$. The precise result, which is due to Bombieri, Francia, Reider and others, can be found in [Ca]:

Theorem 5.5 ([Ca], Theorem 1.11(i)). Let $S$ be a surface of general type. Assume that either

(1) $K_{S}^{2} \geqq 5$ or

(2) $K_{S}^{2} \geqq 2$ and $p_{g} \geqq 1$, but it does not happen that $q=p_{g}=1$ and $K_{S}^{2}=3$ or 4 .

If $n \geqq 2$, then $K_{2}^{\otimes n}$ is base-point-free.

With this we are ready to obtain the following

Corollary 5.6. Let $S$ be a regular surface of general type with ample canonical bundle and $p_{g} \geqq 3$. Then

(1) $H^{1}\left(M_{K_{S}^{\otimes 4+k}} \otimes K_{S}^{\otimes 4+l}\right)=0$ for all $k, l \geqq 0$, and

(2) $H^{1}\left(M_{K_{S}^{\otimes 4+k}}^{\otimes 2} \otimes K_{S}^{\otimes 4+l}\right)=0$ for $k=l=0$, for all $k, l \geqq 1$, and for all $k \geqq 0, l \geqq 2$.

In particular, if $n \geqq 4,\left|K_{S}^{\otimes n}\right|$ embeds $S$ as a projectively normal variety with homogeneous ideal generated by quadratic equations.

Proof. The result is a straightforward consequence of Theorem 5.1 if $p_{g} \geqq 4$, setting $B=K_{\mathrm{S}}^{\otimes 2}$. However we can take advantage of the fact that we are dealing with base-pointfree line bundles of particularly nice shape. If one goes through the steps of the proof of Theorem 5.1, one sees that one of the places when we use $p_{g} \geqq 4$ is to prove that $K_{S} \otimes B$ is base-point-free. In this setting we know this to be true by Theorem 5.5. The other place where we use the bound on $p_{g}$ is when checking the inequalities needed to apply Proposition 2.5 and Proposition 2.4. The reader can see that in this particular case $p_{g} \geqq 3$ suffices for such a purpose.

Remark 5.7. Some hypothesis of Corollary 5.6 can be dropped or relaxed. If we don't require $K_{S}$ to be ample, we obtain essentially the same result: the image of $S$ by $\left|K_{S}^{\otimes n}\right|$ is a projectively normal variety with homogeneous ideal generated by quadratic equations. Indeed, note that the ampleness of $B$ was used in Theorem 5.1 to obtain cohomology vanishings and the base-point-freeness of $K_{S} \otimes B$. Those are taken care now by Theorem 5.5 and Kawamata-Viehweg Vanishing Theorem. On the other hand we can relax the hypothesis on $p_{g}$ to obtain a weaker result, proven by the same techniques: 
(5.7.1) Let $S$ be a regular surface of general type with either $p_{g} \geqq 1$ and $K_{S}^{2} \geqq 2$ or $K_{S}^{2} \geqq 5$. If $n \geqq 5$, then the image of $S$ by the complete linear series $\left|K_{S}^{\otimes n}\right|$ is a projectively normal variety.

We now complete the picture with the nonzero irregularity case:

Theorem 5.8. Let $S$ be an irregular surface of positive Kodaira dimension. Let $B$ be an ample line bundle such that $B^{2} \geqq 5$ and $B^{\prime}$ is base-point-free and $H^{1}\left(B^{\prime}\right)=0$ for all $B^{\prime}$ homologous to $B$ (respectively numerically equivalent). Let L homologous to $K \otimes B^{\otimes n}$ (respectively numerically equivalent) and $L^{\prime}$ homologous to $K \otimes B^{\otimes I}$ (respectively numerically equivalent).

(1) If $\kappa(S)=1$ and $B^{2}>K_{S} \cdot B$, and if $n, l \geqq 2$, then $H^{1}\left(M_{L} \otimes L^{\prime}\right)=0$; if $n, l \geqq 3$, then $H^{1}\left(M_{L}^{\otimes 2} \otimes L^{\prime}\right)=0$. In particular L satisfies property $N_{0}$ if $n \geqq 2$, and L satisfies property $N_{1}$ if $n \geqq 3$.

(2) If $\kappa(S)=2$ and $B^{2} \geqq 2 K_{S} \cdot B$, and if $n, l \geqq 2$, then $H^{1}\left(M_{L} \otimes L^{\prime}\right)=0$. In particular, $L$ satisfies property $N_{0}$ if $n \geqq 2$.

(3) If $\kappa(S)=2$ and $B^{2} \geqq K_{S} \cdot B$, and if $n, l \geqq 3$, then

$$
H^{1}\left(M_{L} \otimes L^{\prime}\right)=H^{1}\left(M_{L}^{\otimes 2} \otimes L^{\prime}\right)=0 .
$$

In particular, L satisfies property $N_{1}$ if $n \geqq 3$.

Sketch of proof. The proof uses Lemma 5.2, the intersection number inequalities in our hypothesis and arguments similar to those in Section 4. We will outline the argument to show (1). Assume for simplicity's sake that $L=B^{\otimes n}$. The vanishing of $H^{1}\left(M_{L} \otimes L^{\prime}\right)=0$ is equivalent (because of Kodaira Vanishing Theorem) to the surjectivity of

$$
H^{0}(L) \otimes H^{0}\left(L^{\prime}\right) \rightarrow H^{0}\left(L \otimes L^{\prime}\right)
$$

Now we break up $L^{\prime}$ as the tensor product $B_{1} \otimes B_{2} \otimes \cdots \otimes B_{l-1} \otimes\left(K_{S} \otimes B_{l}\right)$ where $B_{1}=B \otimes E$ where $E \in \operatorname{Pic}^{0}(S)$ and $E^{\otimes 2} \neq \mathcal{O}$. Clearly, if $L^{\prime}$ is homologous to $K \otimes B^{\otimes l}, B_{i}$ is homologous to $B$ and in any case numerically equivalent, hence by hypothesis and Lemma 2.13, $B_{i}$ is base-point-free and so is $K_{S} \otimes B_{l}$. By Observation 1.4.1 it would suffice to show the surjectivity of several multiplication maps. The first one would be

$$
H^{0}(L) \otimes H^{0}\left(B_{1}\right) \rightarrow H^{0}\left(L \otimes B_{1}\right)
$$

This follows from (1.4.2), the required vanishings being obtained from Kodaira Vanishing Theorem and, if $n=2$, from our choice of $E$. The possible intermediate maps are surjective by (1.4.2) and Kodaira. The last of the maps is surjective by (1.4.2), Kodaira Vanishing Theorem and our hypothesis. Indeed, the required vanishings of $H^{1}$ follow from Lemma 5.2 (here we use the condition $B^{2}>B \cdot K_{S}$ ), using the divisibility of $\mathrm{Pic}^{0}$. We also need the vanishings of $H^{2}(N)$, where $N \equiv B^{\otimes k} \otimes K_{S}^{*}$ and $k \geqq 1$ which certainly occur because $S$ is an elliptic surface.

12 Journal für Mathematik. Band 506 
Now we prove $H^{1}\left(M_{L}^{\otimes 2} \otimes L^{\prime}\right)=0$ under the hypothesis in (1). This vanishing is equivalent to the surjectivity of

$$
H^{0}\left(M_{L} \otimes L^{\prime}\right) \otimes H^{0}(L) \rightarrow H^{0}\left(M_{L} \otimes L \otimes L^{\prime}\right)
$$

by virtue of the vanishing just shown. If we break $L$ as tensor product of $K_{S}$ and base-pointfree line bundles homologous or numerically equivalent to $B$ depending on the case, we can use Observation 1.4.1 and (1.4.2). The vanishings we need to check have already been shown in the first part of the proof.

Corollary 5.9. Let $S$ be an irregular surface of general type with $K_{S}^{2} \geqq 5$. If $n \geqq 5$, then the image of $S$ by the complete linear series $\left|K_{S}^{\otimes n}\right|$ is a projectively normal variety.

Proof. The observation about ampleness made in Remark 5.7 also applies here. Having that in account, the result follows from the arguments of the proof of Theorem 5.8 if $n$ is odd, and for $n$ even we argue as in Corollary 2.15.

Another quite interesting consequence of Theorems 5.1 and 5.8, and of a result by Fernández del Busto is the following effective bound along the lines of Mukai's conjecture:

Corollary 5.10. Let $S$ be an algebraic surface of positive Kodaira dimension, let $A$ be an ample line bundle and let $m=\left[\frac{\left(A \cdot\left(K_{S}+4 A\right)+1\right)^{2}}{2 A^{2}}\right]$. Let $L=K_{S} \otimes A^{\otimes n}$. If $n \geqq 2 m$, then $L$ satisfies property $N_{0}$. If $n \geqq 3 m$, then $L$ satisfies property $N_{1}$. If $S$ is a regular surface of general type and $n \geqq 2 m$, then $L$ satisfies property $N_{1}$.

Sketch of proof. The key observation is the fact that if $k \geqq m$ then it follows from [FdB], Section 2 that $A^{\otimes m}$ is base-point-free and $H^{1}\left(A^{\otimes m}\right)=0$. Then we take $A^{\otimes m}$ as the base-point-free line bundle $B$ in Theorems 5.1 and 5.8. One can easily verify that the numerical conditions in the statements are satisfied. Note that $\operatorname{Pic}^{0}(S)$ is divisible, so if $S$ is irregular, Fernández del Busto's result applies also to $B^{\prime}$ in the statement of Theorem 5.8. There is however one hypothesis of Theorem 5.1 which we have not assumed in this corollary, and which in fact does not occur in general under the hypothesis of our statement. That is the assumption of $p_{g} \geqq 4$. This hypothesis was used in the proof of Theorem 5.1 to check the inequalities needed to apply Proposition 2.4 or Proposition 2.5. Under our current hypothesis $B^{2}$ is much larger than $K_{S} \cdot B$ and in any case, large enough to render the mentioned assumption unnecessary (see for instance (5.4.1)). Therefore the theorem is either a direct consequence of Theorems 5.1 and 5.8 or follows from slight modifications of the arguments involved in proving those theorems, for we are in a situation similar to Corollary 2.15 .

Remark 5.10.1. With the notation of Corollary 5.10, using the above methods one can show that if $X$ is a regular surface of positive Kodaira dimension then $L$ satisfies $N_{1}$ for all $n \geqq m+3$.

We focus now on the study of higher syzygies of pluricanonical models of surfaces of general type. Recall that one can obtain a result regarding them from Theorem 1.3: 
Corollary 5.11. Let $S$ be a surface of general type satisfying the assumptions of Theorem 5.5. If $n \geqq 2 p+4$, then the image of $S$ by $\left|K_{S}^{\otimes n}\right|$ is projectively normal, its ideal is generated by quadrics and the resolution of its homogeneous coordinate ring is linear until the pth stage.

Proof. The line bundle $K_{S}^{\otimes 2}$ is base-point-free by Theorem 5.5. On the other hand, $K_{S}^{\otimes 2}$ is 3-regular by the Kawamata-Viehweg Vanishing Theorem. Hence from Theorem 1.3 the result follows for $n$ even. If $n$ is odd we argue as in Corollory 2.15, writing $K_{S}^{\otimes n}$ as $B^{\otimes s-1} \otimes B^{\prime}$, where $B=K_{S}^{\otimes 2}$ and $B^{\prime}=K_{S}^{\otimes 3}$, which are base-point-free by Theorem 5.5.

This result can be improved for regular surfaces if we impose the hypothesis of Corollary 5.6:

Theorem 5.12. Let $S$ be a regular surface of general type with $p_{g} \geqq 3$. Let

$$
L=K_{S}^{\otimes 2 p+2+l}
$$

and $L^{\prime}=K_{S}^{\otimes 2 p+2+k}$.

(1) If $p \geqq 2, H^{1}\left(M_{L}^{\otimes p+1} \otimes L^{\prime}\right)=0$ for all $k, l \geqq 0$, and

(2) if $p=1, H^{1}\left(M_{L}^{\otimes p+1} \otimes L^{\prime}\right)=0, k=l=0$ for all $k, l \geqq 1$, and for all $k \geqq 0, l \geqq 2$.

In particular, if $n \geqq 2 p+2$ and $p \geqq 1$, then the image of $S$ by $\left|K_{S}^{\otimes n}\right|$ is projectively normal, its ideal is generated by quadrics and the resolution of its homogeneous coordinate ring is linear until the pth stage.

Proof. The proof is by induction on $p$. The statement for $p=1$ is Corollary 5.6 (2), having in account the observation on ampleness made in Remark 5.7. Let us assume the result to be true for $p-1$ and prove the vanishing for $p$. Tensoring (1.1) with $M_{L}^{\otimes p} \otimes L^{\prime}$ and taking global sections yields the following long exact sequence

$$
\begin{gathered}
H^{0}(L) \otimes H^{0}\left(M_{L}^{\otimes p} \otimes L^{\prime}\right) \stackrel{\eta}{\longrightarrow} H^{0}\left(M_{L} \otimes L \otimes L^{\prime}\right) \\
\longrightarrow H^{1}\left(M_{L}^{\otimes p} \otimes L^{\prime}\right) \longrightarrow H^{0}(L) \otimes H^{1}\left(M_{L}^{\otimes p+1} \otimes L^{\prime}\right)
\end{gathered}
$$

The last term is zero by induction assumption, thus the vanishing is equivalent to showing the surjectivity of the multiplication map $\eta$. Let $B=K_{S}^{\otimes 2}$ and $B^{\prime}=K_{S}^{\otimes 3}$. By Observation 1.4.1 it suffices to show the surjectivity of several maps:

$$
H^{0}(B) \otimes H^{0}\left(M_{L}^{\otimes p} \otimes L^{\prime}\right) \stackrel{\eta^{\prime}}{\longrightarrow} H^{0}\left(M_{L} \otimes\left(B \otimes L^{\prime}\right)\right), \quad \text { for all } l, k \geqq 0
$$

and,

$$
H^{0}\left(B^{\prime}\right) \otimes H^{0}\left(M_{L}^{\otimes p} \otimes L^{\prime} \otimes B\right) \stackrel{\eta^{\prime \prime}}{\longrightarrow} H^{0}\left(M_{L} \otimes\left(B \otimes B^{\prime} \otimes L^{\prime}\right)\right), \quad \text { for all } l, k \geqq 0 .
$$

The surjectivity of $\eta^{\prime \prime}$ follows by (1.4.2), the vanishings required following by induction, from (1.1) and Kawamata-Viehweg. The surjectivity of $\eta^{\prime}$ also follows from (1.4.2) by the 
same reasons, but as in the proof of Theorem 2.14 we could alternatively argue restricting to a smooth curve $C$ in $|B|$. We would have to eventually use Proposition 2.4 and the fact that the needed inequalities hold follows from adjunction and the assumption on $p_{g}$, having in account that $h^{1}\left(B \otimes \mathcal{O}_{C}\right)=h^{2}\left(\mathcal{O}_{S}\right)$.

Finally, the statement on the coordinate ring of the image of the pluricanonical maps follows from Corollary 5.6, the vanishings just proven and Theorem 1.2.

As a corollary of Theorem 5.1 (4) and Del Busto's result we obtain an effective bound for a power of an ample line bundle to satisfy property $N_{p}$ :

Corollary 5.13. Let $S$ be a regular surface of general type, let $A$ be an ample line bundle and let $m$ be as in Corollary 5.10. Let $L=A^{\otimes n}$. If $n \geqq m p+m$, then $L$ satisfies property $N_{p}$.

We state now a result for normal presentation and Koszul property of adjoint linear series on regular surfaces of general type with base-point-free canonical bundle:

Theorem 5.14. Let $S$ be a regular surface of general type with $p_{g} \geqq 4$ and base-point-free canonical bundle. Let $B$ be an ample and base-point-free line bundle on $S$ with $H^{1}(B)=0$ and let $B^{2} \geqq B \cdot K_{S}$. Let $L=K_{S} \otimes B^{\otimes n}$ and $L^{\prime}=K_{S} \otimes B^{\otimes m}$. If $n, m \geqq 2$, then

$$
H^{1}\left(M_{L} \otimes L^{\prime}\right)=H^{1}\left(M_{L}^{\otimes 2} \otimes L^{\prime}\right)=0 .
$$

In particular, if $n \geqq 2$, then $K_{S} \otimes B^{\otimes n}$ satisfies property $N_{1}$, and in addition, the Koszul property.

Proof. Cohomology vanishings: First we check the vanishing of $H^{1}\left(M_{L} \otimes L^{\prime}\right)$. By Kodaira Vanishing Theorem, it suffices to check the surjectivity of

$$
H^{0}\left(L^{\prime}\right) \otimes H^{0}(L) \rightarrow H^{0}\left(L \otimes L^{\prime}\right) .
$$

Recall that both $B$ and $K_{S}$ are base-point-free. By Observation 1.4.1 it suffices to check the surjectivity of

$$
\begin{aligned}
H^{0}\left(K_{S} \otimes B^{\otimes n}\right) \otimes H^{0}(B) \stackrel{\alpha}{\longrightarrow} H^{0}\left(K_{S} \otimes B^{\otimes n+1}\right) & \text { for all } n \geqq 2, \\
H^{0}\left(K_{S} \otimes B^{\otimes m}\right) \otimes H^{0}\left(K_{S}\right) \stackrel{\beta}{\longrightarrow} H^{0}\left(K_{S}^{\otimes 2} \otimes B^{\otimes m}\right) & \text { for all } m \geqq 4 .
\end{aligned}
$$

Let $C$ be a smooth curve in $\left|K_{S}\right|$. The surjectivity of $\beta$ follows by (1.4.2), Lemma 5.2 and the inequality $B^{2} \geqq K_{\mathrm{S}} \cdot B$. To see the surjectivity of $\alpha$, let us set $G=K_{\mathrm{S}} \otimes B^{\otimes n} \otimes \mathcal{O}_{\mathrm{C}}$. Using Observation 2.3 it suffices to show the surjectivity of

$$
H^{0}(G) \otimes H^{0}\left(B \otimes \mathcal{O}_{C}\right) \stackrel{\gamma}{\longrightarrow} H^{0}(G \otimes B)
$$

Note that $\operatorname{deg} G+\operatorname{deg}\left(B \otimes \mathcal{O}_{C}\right) \geqq K_{S} \cdot B+3 B^{2} \geqq 2\left(K_{S} \cdot B+B^{2}\right)=4 g(C)-4$. Since by the inequality $B^{2} \geqq K_{S} \cdot B$ and the ampleness of $B, h^{1}\left(B \otimes \mathcal{O}_{C}\right)=p_{g}$ or $p_{g}-1$, and $p_{g} \geqq 4$, the surjectivity of $\gamma$ follows from Proposition 2.5. 
To prove the vanishing of $H^{1}\left(M_{L}^{\otimes 2} \otimes L^{\prime}\right)$, by the vanishing just proved, it suffices to see the surjectivity of

$$
H^{0}\left(M_{L} \otimes L^{\prime}\right) \otimes H^{0}(L) \rightarrow H^{0}\left(M_{L} \otimes L \otimes L^{\prime}\right)
$$

Using Observation 1.4.1 it is enough to prove the surjectivity of

$$
\begin{gathered}
H^{0}\left(M_{L} \otimes L^{\prime}\right) \otimes H^{0}(B) \rightarrow H^{0}\left(M_{L} \otimes \otimes L^{\prime} \otimes B\right), \\
H^{0}\left(M_{L} \otimes L^{\prime} \otimes B^{\otimes 2}\right) \otimes H^{0}\left(K_{S}\right) \rightarrow H^{0}\left(M_{L} \otimes L^{\prime} \otimes K_{S} \otimes B^{\otimes 2}\right) .
\end{gathered}
$$

The surjectivity of the second family of maps follows by (1.4.2) and the same arguments used for the cohomology vanishing already proven. For the first family we argue restricting to $C$. By Observation 2.3, Lemma 2.9, and having in account the already proven surjectivity of $\gamma$, we see that it suffices to check the surjectivity of

$$
H^{0}\left(M_{N} \otimes N^{\prime}\right) \otimes H^{0}\left(B \otimes \mathcal{O}_{C}\right) \stackrel{\delta}{\longrightarrow} H^{0}\left(M_{N} \otimes B \otimes N^{\prime}\right)
$$

where $N=L \otimes \mathcal{O}_{C}$ and $N^{\prime}=L^{\prime} \otimes \mathcal{O}_{C}$. Now $\operatorname{deg} G \geqq 2 g(C)-2+B^{2}$ and $B^{2} \geqq K_{S}^{2} \geqq 4$ by Lemma 5.3 and Nöther's inequality, hence $M_{G}$ is semistable. Then $\delta$ surjects by Proposition 2.4.

Koszul: According to Lemma 3.2 we need to show that $M^{h, L}$ is globally generated and that

$$
H^{0}\left(M^{h, L}\right) \otimes H^{0}\left(L^{\otimes s}\right) \stackrel{\alpha}{\longrightarrow} H^{0}\left(M^{h, L} \otimes L^{\otimes s}\right)
$$

surjects for all $h \geqq 0$ and $s \geqq 1$. Let $B^{\prime}=K_{S} \otimes B$, ample and base-point-free by Lemma 2.13. By Observation 1.4.1, it suffices to prove the surjectivity of

$$
\begin{gathered}
H^{0}\left(M^{h, L} \otimes B^{\otimes l}\right) \otimes H^{0}(B) \stackrel{\beta_{l}}{\longrightarrow} H^{0}\left(M^{h, L} \otimes B^{\otimes l+1}\right) \quad \text { for all } l \geqq 0, \\
H^{0}\left(M^{h, L} \otimes B^{\otimes k} \otimes B^{\prime \otimes r}\right) \otimes H^{0}\left(B^{\prime}\right) \stackrel{\gamma_{r}}{\longrightarrow} H^{0}\left(M^{h, L} \otimes B^{\otimes k} \otimes B^{\prime \otimes r+1}\right) \text { for all } k \geqq 1, r \geqq 0 .
\end{gathered}
$$

We explain in some detail one of the border cases:

$$
H^{0}\left(M^{h, L}\right) \otimes H^{0}(B) \stackrel{\beta}{\longrightarrow} H^{0}\left(M^{h, L} \otimes B\right)
$$

and leave the others to the reader. The proof of the surjectivity of $\beta$ goes by induction and as in Theorem 3.5, it is convenient to prove the vanishing of $H^{1}\left(M^{h, L} \otimes B^{*}\right)$ at the same time. If $h=0$, the surjectivity follows from the arguments sketched in the first part of the proof. Assume the statement to be true for $h-1$. Consider the sequence

$$
\begin{gathered}
H^{0}\left(M^{h-1, L}\right) \otimes H^{0}\left(L \otimes B^{*}\right) \stackrel{\delta}{\longrightarrow} H^{0}\left(M^{h-1, L} \otimes L \otimes B^{*}\right) \\
\longrightarrow H^{1}\left(M^{h, L} \otimes B^{*}\right) \longrightarrow H^{1}\left(M^{h-1, L}\right) \otimes H^{0}\left(L \otimes B^{*}\right) .
\end{gathered}
$$


The multiplication map $\delta$ is surjective by induction hypothesis. The group $H^{1}\left(M^{h-1, L}\right)$ vanishes also by induction hypothesis, therefore $H^{1}\left(M^{h, L} \otimes B^{*}\right)=0$. Now since $H^{1}\left(\mathcal{O}_{X}\right)=0$, in order to see the surjectivity of $\beta$ we proceed as in Theorem $3.5, B$ playing the role $B_{1}$ plays there and restricting to a smooth curve $C \in|B|$, which plays the same role as $b_{1}$. In order to obtain the inequalities needed to apply Lemma 2.10 and Proposition 2.4 , note that $K_{S} \otimes B \otimes \mathcal{O}_{C}=K_{C}$ and recall that $B^{2} \geqq B \cdot K_{S}$ and $\operatorname{deg}\left(K_{S} \otimes \mathcal{O}_{C}\right)=K_{S}^{2} \geqq 4$ by Noether's formula.

As a corollary we obtain an improvement on another result by Ciliberto (cf. [Ci]). As in Remark 5.7 and Corollary 5.9 the hypothesis on the ampleness of $K_{S}$ can be removed, and we state the corollary without it:

Corollary 5.15. Let $S$ be a regular surface of general type with $K_{S}$ base-point-free. Let $p_{g} \geqq 4$. Let $L=K_{S}^{\otimes p+2+l}$. Then, if $p \geqq 1$, the image of $S$ by $|L|$ is projectively normal, its ideal is generated by quadratic equations and the homogeneous coordinate ring is Koszul.

We end up the section with a generalization to higher syzygies of Corollory 5.15:

Theorem 5.16. Let $S$ be a regular surface of general type with $K_{S}$ base-point-free. Let $p_{g} \geqq 4$. Let $L=K_{S}^{\otimes p+2+l}$ and $L^{\prime}=K_{S}^{\otimes p+2+k}$. Then, if $p \geqq 1$,

$$
H^{1}\left(M_{L} \otimes L^{\prime}\right)=H^{1}\left(M_{L}^{\otimes p+1} \otimes L^{\prime}\right)=0 \quad \text { for all } k, l \geqq 0 .
$$

Moreover, if $p \geqq 1$ the image of $S$ by $|L|$ is projectively normal, its ideal is generated by quadratic equations and the resolution of the homogeneous coordinate ring is linear until the pth stage.

Sketch of proof. If $K_{S}$ is ample, the vanishings of $H^{1}\left(M_{L} \otimes L^{\prime}\right)$ and $H^{1}\left(M_{L}^{\otimes 2} \otimes L^{\prime}\right)$ follow from Theorem 5.14 and if $K_{S}$ is not ample, they follow by the same reasoning used for Theorem 5.14, arguing as in Remark 5.7. The proof for $p>1$ follows now by induction. We argue as in the proof of Theorem 5.12. We use Observation 1.4.1, Observation 2.3, Lemma 2.9, and Proposition 2.4 in similar fashion.

Lastly, the statement about the syzygies of the resolution of the pluricanonical models follows from Theorem 1.2 and from the vanishings just proven.

\section{Appendix. Singular surfaces}

We want to point out that the main theorems proved so far in this article also go through for normal surfaces. On smooth surfaces Mukai's conjecture is a natural generalization of the results of Reider on base point freeness and very ampleness to higher syzygies. Recently Kawachi, Maşek and Sakai have extended Reider's theorem to normal surfaces and it is natural to ask for higher syzygy results. Even though there are no Mukai type conjectures on normal surfaces, our results yield Mukai type bounds for higher syzygies. In fact, the bounds obtained for property $N_{p}$ on smooth surfaces also hold for normal surfaces and, in particular, results (1) to (4) in the introduction, remain true. 
More precisely, let $X$ be a surface with canonical singularities. Then Theorems 2.2, 2.11, 2.14, 3.5, 4.1, 4.7 (for surfaces with $K_{X} \equiv 0$ ), and Theorems 5.1, 5.8, 5.12, 5.14, 5.16 (for surfaces of positive Kodaira dimension) hold for $X$ since the same proofs go through. Indeed the delicate points to keep in mind when extending our arguments to the singular case are the use of Kodaira vanishing, Bertini's theorem and the Reider's theorem. The first two hold for surfaces with canonical singularities. The role of the third is played by the results of Kawachi and Maşek (cf. [KM]) and Sakai (cf. [Sa]). Accordingly, Corollaries $2.8,2.12,2.15,4.4,4.5,5.6,5.9,5.11$ and 5.15 follow from the corresponding theorems and $[\mathrm{KM}]$. Finally, Theorems $2.2,2.11,2.14,3.5,4.1,4.7$ and weaker versions of Theorems 5.1 and 5.8 also go through for surfaces with at worst log terminal singularities.

\section{References}

[Bo] E. Bombieri, Canonical models of surfaces of general type, Inst. Hautes Ét. Sci. Publ. Math. 42 (1973), 171-219.

[Bu] D. Butler, Normal generation of vector bundles over a curve, J. Diff. Geom. 39 (1994), 1-34.

[Ca] F. Catanese, Canonical rings and special surfaces of general type, Proceedings of the Summer Research Institute on Algebraic Geometry at Bowdoin 1985, Part 1, AMS (1987), 175-194.

[Ci] C. Ciliberto, Sul grado dei generatori dell'anello canonico di una superficie di tipo generale, Rend. Sem. Mat. Univ. Politecn. Torino 41 (1983), 83-111.

[CD] F.R. Cossec and I.V. Dolgachev, Enriques Surfaces I, Birkhäuser, 1989.

[EL] L. Ein and R. Lazarsfeld, Koszul cohomology and syzygies of projective varieties, Inv. Math. 111 (1993), 51-67.

[EKS] D. Eisenbud, J. Koh, M. Stillman, Determinantal equations for curves of high degree, Amer. J. Math. 110 (1988), 513-539.

[FdB] G. Fernández del Busto, A Matsusaka-type theorem on surfaces, J. Alg. Geom. 5 (1996), 513-520.

[FV] $M$. Finkelberg and $A$. Vishik, The coordinate ring of a general curve of genus $g \geqq 5$ is Koszul, J. Algebra 162 (1993), 535-539.

[GP1] F.J. Gallego and B.P. Purnaprajna, Normal presentation on elliptic ruled surfaces, J. Algebra 186 (1996), 597-625.

[GP2] F.J. Gallego and B.P. Purnaprajna, Higher syzygies of elliptic ruled surfaces, J. Algebra 186 (1996), 626-659.

[GP3] F.J. Gallego and B.P. Purnaprajna, Vanishing theorems and syzygies for K 3 surfaces and Fano varieties, J. Pure App. Alg., to appear.

[GP4] F.J. Gallego and B.P. Purnaprajna, Very ampleness and higher syzygies for Calabi-Yau threefolds, Math. Ann. 312 (1998), 133-149.

[G1] M. Green, Koszul cohomology and the geometry of projective varieties, J. Diff. Geom. 19 (1984), 125-171.

[G2] M. Green, Koszul cohomology and the geometry of projective varieties II, J. Diff. Geom. 20 (1984), 279-289.

[G3] M. Green, Koszul cohomology and geometry, Lectures on Riemann Surfaces, World Scientific Press, Singapore (1989), 177-200.

[H1] Y. Homma, Projective normality and the defining equations of ample invertible sheaves on elliptic ruled surfaces with $e \geqq 0$, Natural Science Report, Ochanomizu Univ. 31 (1980), 61-73.

[H2] Y. Homma, Projective normality and the defining equations of an elliptic ruled surface with negative invariant, Natural Science Report, Ochanomizu Univ. 33 (1982), 17-26.

[KM] T. Kawachi and V. Massek, Reider-type theorems on normal surfaces, preprint

[Ka] Y. Kawamata, The cone of curves of algebraic varieties, Ann. Math. (2) 119 (1984), 603-633.

[Ke] G. Kempf, Projective coordinate rings of Abelian varieties, Algebraic Analysis, Geometry and Number Theory, the John Hopkins Univ. Press (1989), 225-235.

[L] R. Lazarsfeld, A sampling of vector bundle techniques in the study of linear series, Lectures on Riemann Surfaces, World Scientific Press, Singapore (1989), 500-559.

[Mi] Y. Miyaoka, The Chern class and Kodaira dimension of a minimal variety, Algebraic Geometry - Sendai 1985, Adv. Stud. Pure Math. 10, North-Holland, Amsterdam (1985), 449-476. 
[Mu] D. Mumford, Varieties defined by quadratic equations, Corso CIME in Questions on Algebraic Varieties, Rome (1970), 30-100.

[P1] G. Pareschi, Koszul algebras associated to adjunction bundles, J. Algebra 157 (1993), 161-169.

[P2] G. Pareschi, Gaussian maps and multiplication maps on certain projective varieties, Comp. Math. 98 (1995), 219-268.

[PP] G. Pareschi and B.P. Purnaprajna, Canonical ring of a curve is Koszul: A simple proof, Ill. J. Math., to appear.

[R] I. Reider, Vector bundles of rank 2 and linear systems on an algebraic surface, Ann. Math. (2) 127 (1988), 309-316.

[Sa] F. Sakai, Reider-Serrano's method on normal surfaces, Algebraic Geometry: Proceedings, L'Aquila 1988, Lect. Notes 1417 (1990), 301-319.

[Sh] V.V.Shokurov, A nonvanishing theorem, Math. USSR-Izv. 26 (1985), 591-604.

Departamento de Álgebra, Facultad de Mathemáticas, Universidad Complutense de Madrid, 28040 Madrid e-mail: gallego@eucmos.sim.ucm.es

405 Snow Hall, Department of Mathematics, University of Kansas, Lawrence, Kansas 66045-2142

e-mail: purna@math.ukans.edu

Eingegangen 17. April 1998 